\title{
Photoassociation spectroscopy of cold alkaline earth atoms near the intercombination line
}

\author{
R. Ciuryło ${ }^{\dagger \S}$, E. Tiesinga ${ }^{\dagger}$, S. Kotochigova ${ }^{\dagger}$, P. S. Julienne ${ }^{\dagger}$ \\ $\dagger$ Atomic Physics Division, National Institute of Standards and Technology, \\ 100 Bureau Drive, Stop 8423, Gaithersburg, Maryland 20899-8423, USA. \\ $\S$ Instytut Fizyki, Uniwersytet Mikołaja Kopernika, Grudziązka 5/7, 87-100 Toruń, Poland.
}

(Dated: October 22, 2018)

\begin{abstract}
The properties of photoassociation (PA) spectra near the intercombination line (the weak transition between ${ }^{1} S_{0}$ and ${ }^{3} P_{1}$ states) of group II atoms are theoretically investigated. As an example we have carried out a calculation for Calcium atoms colliding at ultra low temperatures of $1 \mathrm{mK}, 1 \mu \mathrm{K}$, and $1 \mathrm{nK}$. Unlike in most current photoassociation spectroscopy the Doppler effect can significantly affect the shape of the investigated lines. Spectra are obtained using $\mathrm{Ca}-\mathrm{Ca}$ and $\mathrm{Ca}-\mathrm{Ca}^{*}$ short-range $a b$ initio potentials and long-range van der Waals and resonance dipole potentials. The similar van der Waals coefficients of ground ${ }^{1} S_{0}+{ }^{1} S_{0}$ and excited ${ }^{1} S_{0}+{ }^{3} P_{1}$ states cause the PA to differ greatly from those of strong, allowed transitions with resonant dipole interactions. The density of spectral lines is lower, the Condon points are at relatively short range, and the reflection approximation for the Franck-Condon factors is not applicable, and the spontaneous decay to bound ground-state molecules is efficient. Finally, the possibility of efficient production of cold molecules is discussed.
\end{abstract}

PACS numbers: $34.50 . R k, 34.10 .+\mathrm{x}, 32.80 . \mathrm{Pj}$

\section{INTRODUCTION}

There is a growing interest in the properties of cold alkaline-earth atoms. One of the main reasons for this interest is a possible construction of optical clocks, whose precision might exceed that of the current atomic standard of time[1, 2]. In particular, optical clocks based on an intercombination transition of alkaline-earth atoms are seen as good candidates for the next time standard[3, 4, 5]. Increased accuracy of time standards is, for example, desired for the search for a time-dependent variation of fundamental constants in atomic experiments, which thereby would verify claims based on astrophysical data[6].

The recent observation of Bose-Einstein condensation (BEC) in an Ytterbium gas [7] raises hopes for alkaline earth atoms, which have similar electronic structure. Such achievement would allow a study of cold gasses over a wide range of temperatures. Milli- and microkelvin temperatures are reached by Doppler cooling on the ${ }^{1} S_{0}-{ }^{1} P_{1}$ resonance and the ${ }^{1} S_{0}-^{3} P_{1}$ intercombination line, respectively. Nanokelvin temperatures are typical for Bose condensates and are reached by evaporative cooling.

Another important reason for interest in alkaline-earth atoms is the absence of a nuclear spin in some isotopes. This offers an unique opportunity towards a fundamental study of Doppler cooling. Moreover, isotopes with zero and nonzero nuclear spin allow a comparison of Doppler and sub-Doppler cooling [8, 9]. The description of atom-atom interactions is much simpler for a nuclear spin-less system. In fact, the basic theory of photoassociation in strong laser fields [10, 11] might be easier to confirm in alkaline-earth gases than in alkali-metal gases, where to date most of the research has been done.

Scattering of atoms in ground and excited states lead to a collisional frequency shift that contributes to the error budget of an optical clock. A Bose condensate crucially depends on atom-atom collisions. Photoassociative (PA) spectroscopy $[12,13,14,15,16]$ is one of the most powerful tools to characterize these scattering processes. It was developed after the success of laser cooling of neutral atoms in the 1980's [17].

We will focus our investigation on PA spectroscopy of ultracold alkaline-earth atoms. In the presence of laser light, two colliding ground-state atoms, labelled by the scattering state " $g$ ", absorb a photon forming an excited molecular bound state " $e "[16]$. This process is called photoassociation. The excited state decays to product states " $p$ " leading to detectable loss of atoms from an atomic trap. The variation of the atom loss as a function of laser frequency gives the photoassociation spectrum. The shape of photoassociation lines not only depends on the properties of the colliding atoms but also on the temperature and other conditions in a trap [18].

Phoatoassociation spectra close to the resonance of the ${ }^{1} S_{0}-{ }^{1} P_{1}$ transition in alkaline-earth atoms were theoretically analyzed by Machholm et al. [19, 20] and others [21, 22]. Recently, Degenhardt et al. [23] measured the photoassociation spectra of cold calcium atoms near this transition at $\mathrm{mK}$ temperatures. Takahashi et al. [24] used the photoassociation spectroscopy to determined the scattering length of ${ }^{174} \mathrm{Yb}$.

This paper analyzes properties of photoassociation spectra near the intercombination line, i.e. laser frequencies close to the ${ }^{1} S_{0}-{ }^{3} P_{1}$ transition. A dipole transition between pure singlet and triplet states is forbidden. However, alkaline-earth atom states labelled ${ }^{3} P_{1}$ are not pure triplet states and have a small singlet component. This component mostly comes from mixing with the nearby ${ }^{1} P_{1}$ state and give rise to a weak dipole transition between the ${ }^{1} S_{0}$ and ${ }^{3} P_{1}$ state. Consequently, the ${ }^{1} S_{0}-^{3} P_{1}$ atomic line has a small natural width. As an example we have carried out calculations for calcium.

We describe the shape of photoassociation lines with verysmall natural width and weak laser radiation. The Doppler effect as well as the photon recoil must be taken into account. This is in sharp contrast with the usual treatment of PA [18] in which these two effects are neglected. Secondly, we discuss possible patterns of vibrational levels in photoassociation spectra near the intercombination line. Closecoupled ro-vibrational bound states are obtained using an interaction Hamiltonian, which is based on our electronic- 


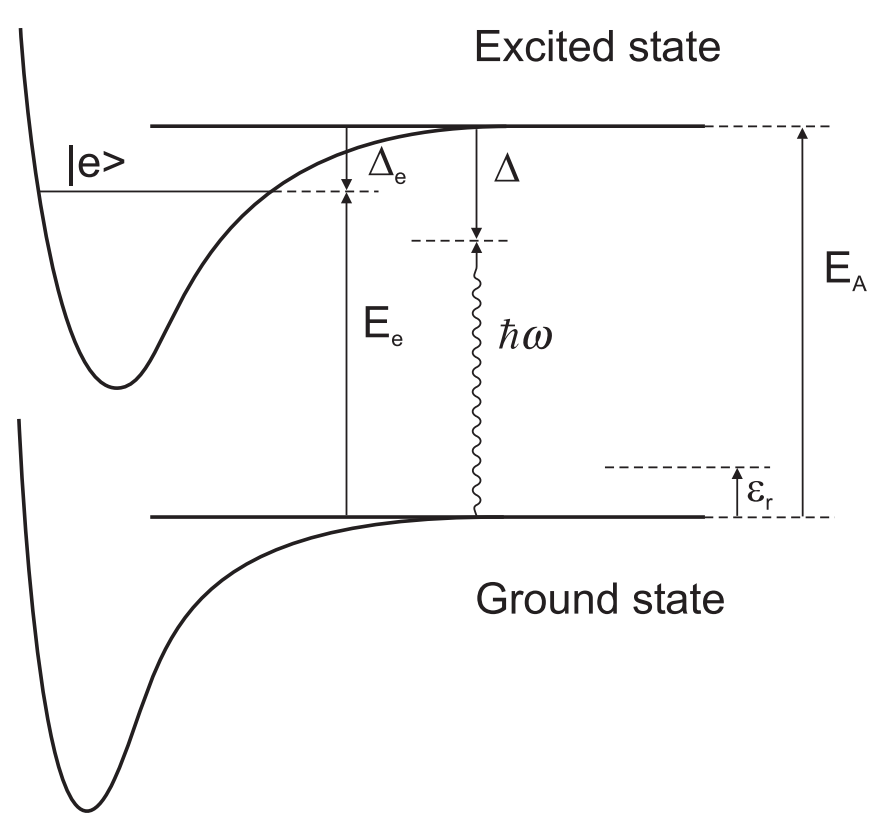

FIG. 1: Energy diagram of the photoassociation process. The zero energy corresponds to separated atoms in the electronic ground state with zero kinetic energy; $E_{A}$ is the energy of an isolated atom in the excited state; $E_{e}$ is the energy of the excited molecular bound state $|e\rangle ; \hbar \omega$ is the photon energy; $\varepsilon_{r}$ is the kinetic energy of the relative motion of colliding atoms; $\Delta_{e}$ is the binding energy of the bound state $|e\rangle ; \Delta$ is the detuning of the photon energy from the isolated atom excitation energy $E_{A}$.

structure potentials 25] and recently calculated dispersion coefficients [26]. The Hamiltonian also includes coupling between ${ }^{1} P_{1}$ and metastable ${ }^{3} P_{0,1,2}$ states. The interatomic potential between two ground state calcium atoms is relatively well known[27]. It is shown that when the interaction in the ground and excited state is similar the reflection approximation [10, 14, 28] cannot be applied to calculate the intensities of photoassociations lines. This is unlike photoassociation spectra near strongly allowed transitions, where the interaction in the ground and excited states differs significantly and the reflection approximation is well satisfied. Finally, we show that efficient production of cold molecules in the ground electronic state using photoassociation should be feasible.

\section{SHAPE OF THE PHOTOASSOCIATION LINE}

The photoassociation process occurs in a thermal cloud of cold atoms at temperature $T$ interacting with weak laser radiation. After absorption of a photon of frequency $\omega$, two atoms form an excited molecular bound state $|e\rangle$ with energy $E_{e}$. The photoassociation process according to standard descriptions is most efficient when the photon energy and the kinetic energy of the relative motion of colliding atoms, $\varepsilon_{r}$, match the energy of the excited bound state, that is $\hbar \omega+\varepsilon_{r}=E_{e}$.

A schematic of energies in the PA process is shown in Fig. 1 The binding energy $\Delta_{e}$ of bound state $|e\rangle$ is given by $\Delta_{e}=E_{e}-E_{A}$, where $E_{A}$ is the energy of an isolated atom
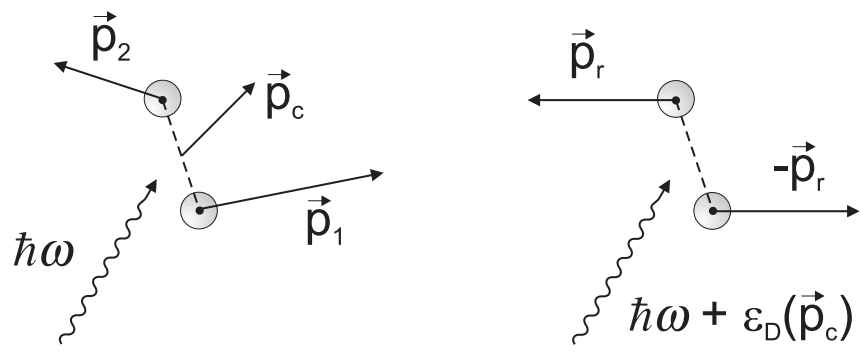

\section{Laboratory coordinates}

\section{Center of mass coordinates}

FIG. 2: Schematic of the collision before the photoassociation process in the laboratory frame and in the center of mass coordinates. $\vec{p}_{1}$ and $\vec{p}_{2}$ are momenta of the colliding atoms; $\vec{p}_{r}$ is the relative momentum of the colliding atoms; $\vec{p}_{c}$ is the center-of-mass momentum of the two atoms in the laboratory frame; $\hbar \omega$ is the photon energy in the laboratory coordinates; $\hbar \omega+\varepsilon_{D}\left(\overrightarrow{p_{c}}\right)$ is the Doppler shifted photon energy in the moving frame of center of mass coordinates.

in the excited state and the zero energy corresponds to separated atoms in the electronic ground state with zero kinetic energy. Furthermore, the detuning $\Delta$ of the photon is defined by $\Delta=\hbar \omega-E_{A}$, where $\hbar \omega$ is the photon energy. The resonance condition for the PA process in terms of detunings reads $\Delta+\varepsilon_{r}-\Delta_{e}=0$.

The description of photoassociation for very narrow lines requires us to include two new effects. These effects are the Doppler shift and the photon recoil. Figure 2 shows a schematic of the collision before the PA process. In the laboratory frame the two colliding atoms, each with mass $m$, have momentum $\vec{p}_{1}$ and $\vec{p}_{2}$, respectively. In the coordinate frame, which moves along with the center of mass, the relative momentum of the colliding atoms is $\vec{p}_{r}$ and kinetic energy of relative motion $\varepsilon_{r}\left(\vec{p}_{r}\right)=p_{r}^{2} / 2 \mu$, where $\mu=m / 2$ is the reduced mass of the colliding atoms. The photon energy $\hbar \omega+\varepsilon_{D}\left(\overrightarrow{p_{c}}\right)$ in the moving frame is shifted with respect to its energy $\hbar \omega$ in the laboratory frame. To good approximation the Doppler shift $\varepsilon_{D}\left(\vec{p}_{c}\right)=-\hbar \vec{k}_{\text {las }} \cdot \vec{p}_{c} / M$ is proportional to $p_{c}$, where $\vec{p}_{c}$ is the center-of-mass momentum of the two atoms in the laboratory frame. The total mass of the system is $M=2 m, \vec{k}_{\text {las }}$ is the wave vector of the laser radiation with absolute value $k_{\text {las }}=\omega / c$, and $c$ is the speed of light. After photoassociation the excited molecule gains the momentum $\hbar \vec{k}_{\text {las }}$ of the absorbed photon and, therefore, has a translational kinetic energy of $E_{\mathrm{rec}, \mathrm{mol}}=\hbar^{2} k_{\text {las }}^{2} /(2 M)$ in the moving frame defined before the absorbtion of the photon. Consequently, photoassociation is most efficient when $\hbar \omega+\varepsilon_{D}\left(\overrightarrow{p_{c}}\right)+\varepsilon_{r}\left(\overrightarrow{p_{r}}\right)=E_{e}+E_{\text {rec,mol }}$ or in terms of detunings $\Delta+\varepsilon_{D}\left(\overrightarrow{p_{c}}\right)+\varepsilon_{r}\left(\overrightarrow{p_{r}}\right)-\Delta_{e}-E_{\mathrm{rec}, \mathrm{mol}}=0$.

The excited molecular states created in the PA process either decay back to the ground state or can be further excited to ionizing states. In the former case the product states escape from the trap and give rise to trap loss. Ions are detected in the latter case. In this paper we will only model trap loss. The loss mechanisms are characterized by a rate coefficient $K(\Delta, T)$, 
which describes the efficiency of the process for a given laser detuning, intensity $I$, and atom temperature. For clarity we omit $I$ as argument in the rate coefficient. The rate coefficient $K(\Delta, T)$ is linear in the weak laser field intensity $I$. For higher intensities the center of the PA line shifts linearly with $I[10,29,30]$ and the width of the line increases. The description of the PA line shape presented in this work, for simplicity, omits this shift as it can be neglected in the weak laser field regime.

The photoassociation trap-loss rate coefficient involves a thermal average of the rate coefficient for a given pair of momenta $\vec{p}_{1}$ and $\vec{p}_{2}$ in the laboratory frame. The momentum distribution of both atoms is Maxwellian with temperature $T$. In practice we average over the momenta $\vec{p}_{c}$ and $\vec{p}_{r}$, denoted by $\langle\ldots\rangle$. These momenta have a Maxwell-Boltzmann distribution $f_{M}\left(\vec{p}_{c}\right)=\left(\sqrt{\pi} p_{M}\right)^{-3} \exp \left(-p_{c}^{2} / p_{M}^{2}\right)$ and $f_{\mu}\left(\vec{p}_{r}\right)=$ $\left(\sqrt{\pi} p_{\mu}\right)^{-3} \exp \left(-p_{r}^{2} / p_{\mu}^{2}\right)$ with the most probable momentum $p_{M}=\sqrt{2 k_{B} T M}$ and $p_{\mu}=\sqrt{2 k_{B} T \mu}$, respectively, where $k_{B}$ is the Boltzmann constant. Then, we have

$$
\begin{aligned}
& K(\Delta, T)=\left\langle\mathcal{K}\left(\Delta, \vec{p}_{c}, \vec{p}_{r}\right)\right\rangle= \\
& \quad \int d^{3} \vec{p}_{c} f_{M}\left(\vec{p}_{c}\right) \int d^{3} \vec{p}_{r} f_{\mu}\left(\vec{p}_{r}\right) \mathcal{K}\left(\Delta, \vec{p}_{c}, \vec{p}_{r}\right),
\end{aligned}
$$

where $\mathcal{K}\left(\Delta, \vec{p}_{c}, \vec{p}_{r}\right)$ describes the trap loss from a collision with momenta $\vec{p}_{c}$ and $\vec{p}_{r}$.

The trap-loss coefficient for $\vec{p}_{c}$ and $\vec{p}_{r}$ is equal to

$$
\mathcal{K}\left(\Delta, \vec{p}_{c}, \vec{p}_{r}\right)=\sum_{e, g} v_{r} \frac{\pi}{k_{r}^{2}}\left|S_{p g}\left(\Delta, \overrightarrow{p_{c}}, \overrightarrow{p_{r}} ; e\right)\right|^{2},
$$

where $k_{r}$ is the relative wavenumber defined by $\varepsilon_{r}=$ $\hbar^{2} k_{r}^{2} /(2 \mu)$ and $v_{r}=\hbar k_{r} / \mu$ is the relative speed of the colliding atoms. The quantity $\left|S_{p g}\left(\Delta, \overrightarrow{p_{c}}, \overrightarrow{p_{r}} ; e\right)\right|^{2}$ is the transition probability from an initial ground state, $g$, to all product states, $p,[66$ ] through an intermediate excited bound state $e$. The indices $g$ and $e$, summed over in Eq. (2), represent quantum numbers that describe the initial and intermediate state, respectively. Each initial state $g$ is labeled by the total angular momentum quantum number $J_{g}$, its projection quantum number $M_{g}$, and the total parity $p_{g}$, while the intermediate rovibrational levels $e$ are labeled by vibrational quantum number $v$, total angular momentum $J_{e}$, projection $M_{e}$ and parity $p_{e}$.

The transition probability from an initial state to the product states can be described by a generalized resonance formula [10, 14, 31]

$$
\begin{aligned}
& \left|S_{p g}\left(\Delta, \overrightarrow{p_{c}}, \overrightarrow{p_{r}} ; e\right)\right|^{2}= \\
& \quad \frac{\Gamma_{p e} \Gamma_{e g}\left(\varepsilon_{r}\right)}{\left(\Delta+\varepsilon_{D}\left(\overrightarrow{p_{c}}\right)+\varepsilon_{r}\left(\overrightarrow{p_{r}}\right)-\Delta_{e}-E_{\mathrm{rec}, \mathrm{mol}}\right)^{2}+\left(\Gamma_{e} / 2\right)^{2}},
\end{aligned}
$$

where the total width of the excited bound state $e, \Gamma_{e}=$ $\Gamma_{e, \text { nat }}+\Gamma_{e, \text { dis }}+\sum_{g} \Gamma_{e g}\left(\varepsilon_{r}\right)$, is the sum of its natural radiative width $\Gamma_{e, \text { nat }}$, the contribution $\Gamma_{e, \text { dis }}$ from predissociation, and the stimulated widths $\Gamma_{e g}\left(\varepsilon_{r}\right)$ caused by the laser coupling between the excited and ground states. The width $\Gamma_{p e}$ describes the decay into product states and is assumed to be equal to the sum of the natural radiative and predissociation width of the bound state.

The stimulated width is proportional to the light intensity and is calculated from Fermi's golden rule [10, 31]

$$
\Gamma_{e g}\left(\varepsilon_{r}\right)=2 \pi\left|\left\langle\Psi_{e}\left(v, J_{e} M_{e} p_{e}\right)\left|V_{\text {las }}\right| \Psi_{g}^{+}\left(\varepsilon_{r}, J_{g} M_{g} p_{g}\right)\right\rangle\right|^{2},
$$

where $\left|\Psi_{e}\left(v, J_{e} M_{e} p_{e}\right)\right\rangle$ is the unit-normalized excited bound state and $\left|\Psi_{g}^{+}\left(\varepsilon_{r}, J_{g} M_{g} p_{g}\right)\right\rangle$ is the energy normalized scattering ground state. The operator $V_{\text {las }}$ describes the coupling between the ground and excited state by laser light. Details of the close-coupled equations [32] that are solved to calculate the bound and scattering states are described in appendix To describe the atom-photon interaction during a collision we adopt the treatment developed by Napolitano et al. [33]. The matrix elements of $V_{\text {las }}$ are given in appendix B

To highlight properties of the photoassociation lines we reduce the thermal average in Eq. (1) to the 2D integral

$$
\begin{aligned}
& \left\langle\frac{\hbar \pi}{\mu k_{r}}\left|S_{p g}\left(\Delta, \overrightarrow{p_{c}}, \overrightarrow{p_{r}} ; e\right)\right|^{2}\right\rangle= \\
& \frac{k_{B} T}{h Q_{T}} \frac{2}{\sqrt{\pi}} \int_{-\infty}^{+\infty} d y e^{-y^{2}} \int_{0}^{\infty} d x x e^{-x^{2}} \mathcal{L}\left(\Delta, y, x^{2}\right),
\end{aligned}
$$

where $y=-\vec{k}_{\text {las }} \cdot \vec{p}_{c} /\left(k_{\text {las }} p_{M}\right)$ and $x=p_{r} / p_{\mu}$ are dimensionless variables similar to those used in the description of pressure and Doppler broadened spectral lines [34, 35], $Q_{T}=\left(2 \pi \mu k_{B} T / h^{2}\right)^{3 / 2}$, and

$$
\begin{aligned}
& \mathcal{L}\left(\Delta, y, x^{2}\right)= \\
& \quad \frac{\Gamma_{p e} \Gamma_{e g}\left(x^{2} \Delta_{T}\right)}{\left(\Delta+y \Delta_{D}+x^{2} \Delta_{T}-\Delta_{e}-E_{\mathrm{rec}, \mathrm{mol}}\right)^{2}+\left(\Gamma_{e} / 2\right)^{2}} .
\end{aligned}
$$

The quantities $\Delta_{T}=k_{B} T$ and $\Delta_{D}=\hbar k_{\text {las }} \sqrt{2 k_{B} T / M}$ are the thermal and Doppler width, respectively.

Three limiting cases of the lineshape, Eq. 5, are of interest. The shape of the line is Lorentzian when $\Gamma_{e}$ is much bigger than $\Delta_{D}, \Delta_{T}$, and $\Gamma_{e g}\left(\varepsilon_{r}\right)$. The denominator of $\mathcal{L}$ can then be pulled out of the integrals leading to a Lorentzian profile with a full-width at half maximum (FWHM) equal to $\Gamma_{e}$. Such a lineshape can be expected for strongly allowed transitions at ultra-low temperatures on the order of nanokelvins, such as exist in Bose condensates [36, 37] 67].

In a second limiting case the shape of the line is a "cutoff exponential". This profile can be obtained when $\Delta_{T}$ is much larger than $\Delta_{D}$ and $\Gamma_{e}$ and the energy dependence of $\Gamma_{e g}\left(\varepsilon_{r}\right)$ is neglected (compare discussion in Ref. [18] where the energy dependence $\Gamma_{e g}\left(\varepsilon_{r}\right) \sim \varepsilon_{r}^{l_{g}+1 / 2}$ describing the Wigner threshold law behavior is considered). The Lorentzian $\mathcal{L}$ can be replaced by a delta-Dirac function with argument $\Delta-\Delta_{e}-E_{\mathrm{rec}, \mathrm{mol}}+x^{2} \Delta_{T}$ and the integrals can be solved analytically. In fact, the profile is proportional to $\theta(-\Delta) \exp \left(\Delta / \Delta_{T}\right)$, where $\theta(z)$ is the Heaviside step function: $\theta(z)=1$ for $z \geq 1$ and $\theta(z)=0$ for $z<1$. The full width at $1 / e$ of the exponential lineshape equals the thermal 
width $\Delta_{T}$. This lineshape is most easily observed at magnetooptical trapping temperatures on the order of a millikelvin.

Finally, for the unusual situation of extremely weak transitions at nanokelvin temperatures one could try to achieve conditions in which $\Delta_{D}$ is much bigger than $\Delta_{T}$ and $\Gamma_{e}$. In such a case the Lorentzian can again be replaced by a delta function but now with an argument that only depends on $y$ and $\Delta$. The resulting lineshape is a Gaussian with half width at $1 / e$ of the maximum equal to $\Delta_{D}$.

In the usual treatment of the PA line shape Doppler broadening is neglected. To find conditions for which this approximation breaks down, the relative importance of Doppler and thermal effects must be determined. It is easy to show that $\Delta_{D}=\Delta_{T}$ at temperature $T=T_{R}$, where $T_{R}=$ $\hbar^{2} k_{\text {las }}^{2} /\left(m k_{B}\right)$ is the atomic recoil temperature. At temperatures $T>T_{R}$ thermal broadening dominates, while for $T<T_{R}$ Doppler broadening can determine the shape of the line. In fact, this requirement is not sufficient. It is also necessary to assume that the Doppler width is comparable or bigger than $\Gamma_{e}$.

The influence of the photon recoil on the PA spectra is to very good approximation described by an uniform shift,

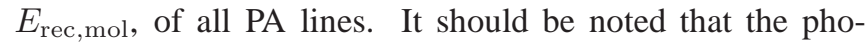
ton recoil energy of the two-atom molecule is two times smaller than the photon recoil energy of an isolated atom $E_{\text {rec }}=\hbar^{2} k_{\text {las }}^{2} /(2 m)$. This indicates that for molecular bound states close to atomic levels there should exist a transition region from recoiling as a molecule to recoiling as an atom. Our theory does not treat this effect and should only be applied to molecular states with a binding energy that is much bigger than the photon recoil energy.

\section{INTERATOMIC HAMILTONIAN}

Properties of photoassociation spectra are governed by the interactions between colliding atoms. The interaction Hamiltonian is described in appendix $\mathrm{A}$ and is similar to that discussed by Mies et al. [39] for the electronic structure and spectroscopy of $\mathrm{Hg}_{2}$. This Hamiltonian includes non-relativistic Born-Oppenheimer potentials, the spin-orbit splitting of the ${ }^{3} P_{0,1,2}$ atomic states, relativistic coupling between ${ }^{1} P$ and ${ }^{3} P$ states, and a term that incorporates the rotation of the two atom system.

The best possible Born-Oppenheimer potentials are used. $A b$ initio calculations, with the exception of a few simple cases, do not give sufficiently accurate predictions of absolute positions of molecular bound states. Therefore theoretical potentials are used as initial guesses, and modified at short interatomic separation to reproduce experimental binding energies. Unfortunately there is no experimental data on binding energies near the ${ }^{3} P_{1}+{ }^{1} S_{0}$ dissociation limit for the calcium molecule. As the rovibrational structure is not known experimentally, we can only map out possible spectra by varying the short-range part of the potential.

The Born-Oppenheimer potentials have Hund's case (a) symmetry, ${ }^{2 S+1}|\Lambda|_{\sigma}$, where $S$ is the total electron spin, $\Lambda$ is the projection of the total electron orbital angular momentum

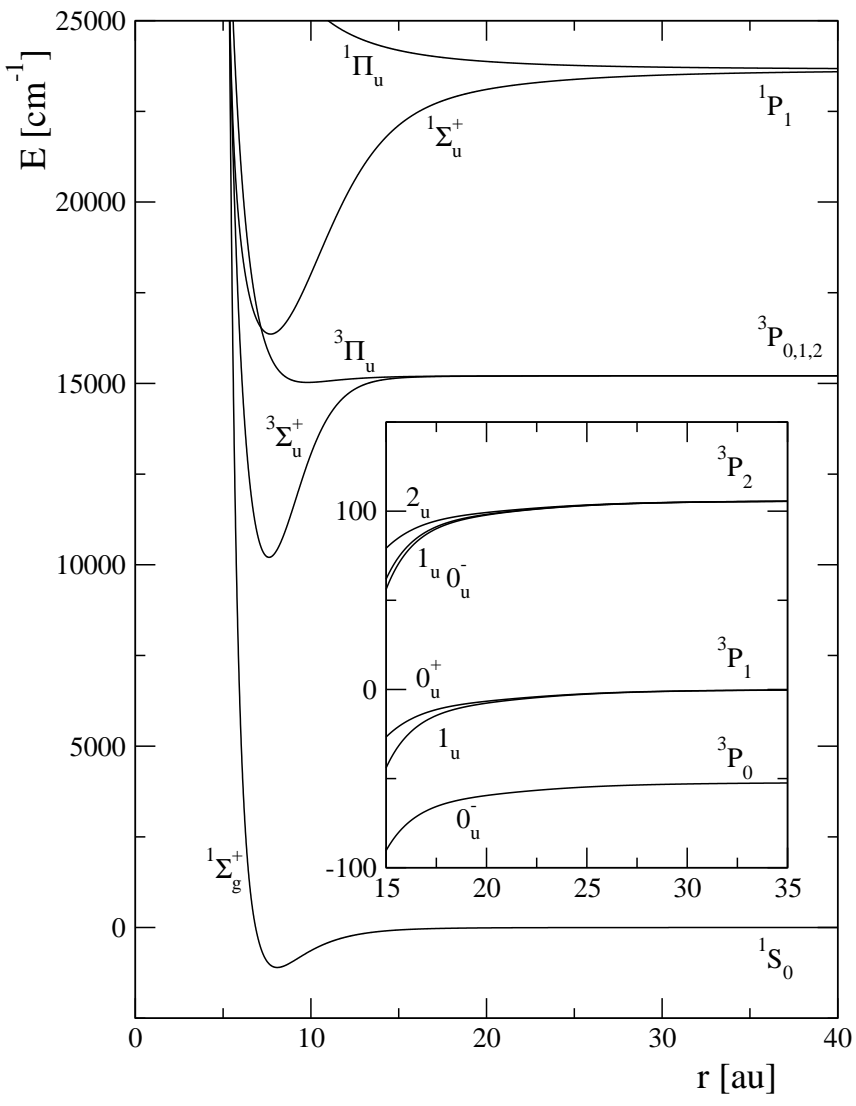

FIG. 3: Adiabatic potentials of two calcium atoms as a function of interatomic separation. The inset shows a blowup of the region near the ${ }^{3} P+{ }^{1} S$ limits. At short range the potentials are labelled by their Hund's case (a) symmetry and in the inset by a Hund's case (c) symmetry label. Ungerade excited states, accessible by an optically allowed transition from the ground $\mathrm{X}^{1} \Sigma_{g}^{+}$potential, are shown.

along the interatomic axis, and $\sigma=g / u$ describes the gerade or ungerade symmetry of a state. Near the ${ }^{3} P_{1}+{ }^{1} S_{0}$ limit, where the atomic spin-orbit interaction is much bigger than the Born-Oppenheimer potentials, adiabatic potentials are better described by Hund's case (c) symmetry, $|\Omega|_{\sigma}^{ \pm}$, where $\Omega$ is the projection of the total electron angular momentum along the interatomic axis and for $\Omega=0$, the label \pm describes the symmetry under a reflection of the electronic wavefunction. The adiabatic Hund's case (c) potentials are obtained by simultaneous diagonalization of the Born-Oppenheimer potentials and the spin-orbit coupling.

Relevant data about the interaction potentials between alkaline-earth atoms in the ground and excited states have been compiled by Kotochigova and Julienne [25]. The $\mathrm{Ca}_{2}$ potentials [25] are shown in Fig. 3. Czuchaj et al. [40] have published a similar set of potentials. It is convenient to use atomic units. The atomic unit of length 1 au $=1 a_{0}=$ $0.0529177 \mathrm{~nm}$ is equal to a Bohr radius $a_{0}=\hbar^{2} /\left(m_{e} e^{2}\right)$; the atomic unit of energy $1 \mathrm{au}=E_{h}=4.3597482 \times 10^{-18} \mathrm{~J}$ is equal to the Hartree energy $E_{h}=\hbar^{2} /\left(m_{e} a_{0}^{2}\right)$. Here $m_{e}$ and $e$ are the electron mass and charge, respectively.

The $X^{1} \Sigma_{g}^{+}$electronic ground state potential of $\mathrm{Ca}_{2}$ has 
been determined by Allard et al.[27]. Parameters of the potential are listed in Table 1 of this reference. For large interatomic separations the potential asymptotically approaches a van der Waals potential with $C_{6}\left(X^{1} \Sigma_{g}^{+}\right)=2081.18 \mathrm{au}$. The atomic unit of $C_{6}$ is $1 \mathrm{au}=E_{h} a_{0}^{6}=0.957342 \times 10^{-79} \mathrm{Jm}^{6}$. The scattering length for this potential is $a_{\text {scat }}=389.8 \mathrm{au}$.

The exited-state interaction potentials between calcium atoms dissociating to the ${ }^{1} P_{1}+{ }^{1} S_{0}$ and ${ }^{3} P_{0,1,2}+{ }^{1} S_{0}$ limits for short-range interatomic separations have been modelled using the adiabatic ${ }^{1,3} \Sigma_{g, u}^{+}$and ${ }^{1,3} \Pi_{g, u}$ potentials. These adiabatic potentials are determined on the basis of ab initio calculations of Ref. [25] and smoothly connected to their asymptotic functional form. The form is $C_{6} / r^{6}$ for triplet states and $C_{3} / r^{3}$ for singlet states, respectively. Triplet potentials dissociate to the ${ }^{3} P+{ }^{1} S$ limits, while singlet potentials dissociate to the ${ }^{1} P+{ }^{1} S$ limit.

In the model describing interaction of two calcium atoms we do not include potentials correlating to ${ }^{1} D_{2}+{ }^{1} S_{0}[41]$ and ${ }^{3} D_{1,2,3}+{ }^{1} S_{0}$ dissociation limits. These potentials could give rise to molecular bound states near the ${ }^{3} P_{1}+{ }^{1} S_{0}$ limit, but the sparse density of states makes it unlikely that such level occur in a small energy interval close to the ${ }^{3} P_{1}+{ }^{1} S_{0}$ dissociation limit.

Dispersion coefficients for two ground state atoms are well known for many atomic species [27, 42, 43]. For excited atoms, however, there is little data. For a calcium atom in the ground ${ }^{1} S$ state interacting with another calcium atom in the excited ${ }^{3} \mathrm{P}$ state the long-range dispersion coefficients have been recently calculated by Derevianko and Porsev[26]. The attractive Hund's case (c) $|\Omega|_{g / u}^{ \pm}$potentials correlating to the ${ }^{3} P_{1}+{ }^{1} S_{0}$ limit have $C_{6}\left(0_{g / u}^{+}\right)=$ 2462 au and $C_{6}\left(1_{g / u}\right)=2593$ au [26]. For this Paper, however, dispersion coefficients are needed for Hund's case (a) ${ }^{2 S+1}|\Lambda|_{g / u}$ Born-Oppenheimer potentials. Following Ref. [39] we have $C_{6}\left(0_{g / u}^{+}\right)=C_{6}\left({ }^{3} \Pi_{g / u}\right), C_{6}\left(1_{g / u}\right)=$ $\left[C_{6}\left({ }^{3} \Sigma_{g / u}\right)+C_{6}\left({ }^{3} \Pi_{g / u}\right)\right] / 2$, and, therefore, $C_{6}\left({ }^{3} \Sigma_{g / u}\right)=$ $2724 \mathrm{au}, C_{6}\left({ }^{3} \Pi_{g / u}\right)=2462$ au. Derevianko and Porsev[26] found that the splitting between the long-range $\Sigma$ and $\Pi$ potentials is small. This small anisotropy for the quasi-twoelectron atom is qualitatively different from analytical predictions for single-electron excited alkali-metal atoms interacting with rare-gas atoms [44, 45]. Older data [46] does not agree with the results of Ref. [26].

The lifetime of the atomic ${ }^{1} P_{1}$ state determines the dipoledipole interaction coefficients, $C_{3}$, of the singlet potentials. In fact, $C_{3}\left({ }^{1}|\Lambda|_{\sigma}\right)=\zeta\left({ }^{1}|\Lambda|_{\sigma}\right) C_{3}^{(0)}$ where $C_{3}^{(0)}=$ $\left.(3 / 4) \Gamma_{A}\left({ }^{1} P_{1}\right)\left[\lambda_{A}\left({ }^{1} P_{1}\right) /(2 \pi)\right]\right]^{3}, \Gamma_{A}\left({ }^{1} P_{1}\right)=\hbar / \tau_{A}\left({ }^{1} P_{1}\right)$ is the natural width of the excited ${ }^{1} P_{1}$ state, and $\lambda_{A}$ is the wavelength of the corresponding radiation. The coefficient $\zeta\left({ }^{1}|\Lambda|_{\sigma}\right)$ is defined by $\zeta\left({ }^{1} \Sigma_{g}\right)=+2, \zeta\left({ }^{1} \Sigma_{u}\right)=-2$, $\zeta\left({ }^{1} \Pi_{g}\right)=-1$, and $\zeta\left({ }^{1} \Pi_{u}\right)=+1$. For calcium $\tau_{A}\left({ }^{1} P_{1}\right)=$ $4.59 \mathrm{~ns}$ [19, 47].

Retardation effects 48, 49] do not change our main conclusion. The calcium bound states of interest are mostly confined to interatomic separations that are small compared to the wavelength $\lambda_{A}\left({ }^{1} P_{1}\right)$. Under such circumstances retardation effects can be neglected. Moreover, this implies that pho-

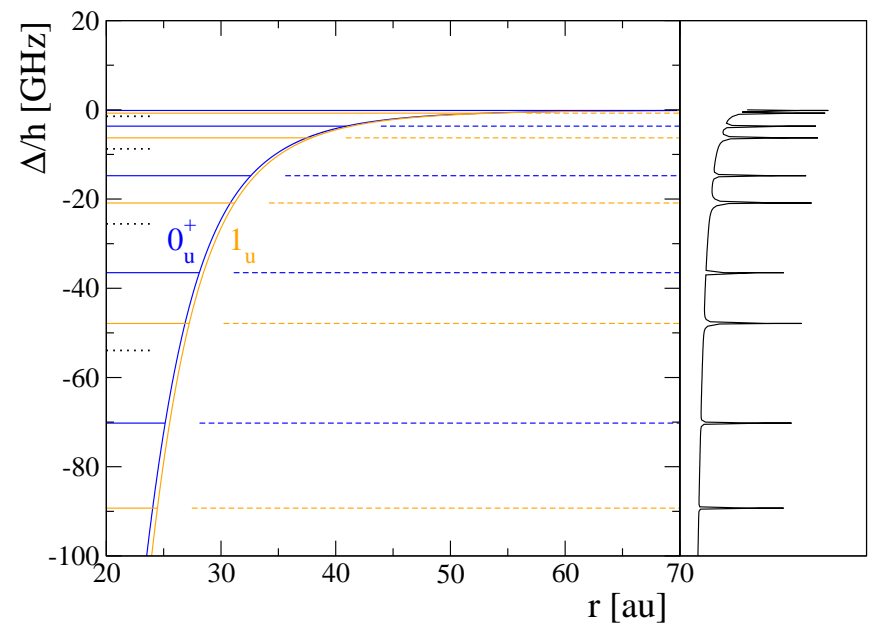

FIG. 4: An example bound-state structure and corresponding photoassociation spectrum of calcium near the intercombination line. The detuning $\Delta$ is defined relative to the ${ }^{3} P_{1}+{ }^{1} S_{0}$ limit. The panel on the left shows the $0_{u}^{+}$(dark line) and $1_{u}$ (light line) Hund's case (c) adiabatic potentials as a function of interatomic separation $r$. The bound states of these potentials are indicated by the thick and thin solid horizontal lines, respectively. The panel on the right shows the corresponding photoassociation spectrum under typical conditions. Each peak corresponds to a bound state in the left panel as indicated by the dashed lines. Finally, the short dotted lines on the left side of the graph indicate "bin edges" of the $0_{u}^{+}$potential.

toassociation of two ground state atoms can only excite the ungerade states. Therefore, Fig. 3 only shows ungerade excited potential curves.

\section{RESULTS}

Photoassociation spectra near the ${ }^{3} P_{1}+{ }^{1} S_{0}$ limit are expected to be weak because the atomic transition dipole to the intercombination line is nearly forbidden. As we will show, such spectra can most easily be measured at ultracold temperatures on the order of $\mu \mathrm{K}$ and below. At these temperatures only $s$-wave collisions will contribute to the spectrum. Consequently, contributing transitions are between the ground gerade scattering state, $\left|\Psi_{g}^{+}\left(\varepsilon_{r}, J_{g} M_{g} p_{g}\right)\right\rangle$, with total molecular angular momentum $J_{g}=l_{g}=0$ and parity $p_{g}=1$, where $l_{g}$ is the rotational angular momentum between the atoms, and excited ungerade bound states $\left|\Psi_{e}\left(v, J_{e} M_{e} p_{e}\right)\right\rangle$ with $J_{e}=1$ and $p_{e}=-1$. For total molecular angular momentum $J_{g}=0$ and $J_{e}=1$ there are one and five coupled channels, respectively (See Appendix $₫$ ). The excited-state channels for odd $J_{e}$ and negative total parity $p_{e}$ do not include the ${ }^{3} P_{0}$ atomic state and, therefore, the predissociation width $\Gamma_{e, \text { dis }}$ in Eq. 4 is zero [68].

Figure 4 shows an example of a bound state structure and the corresponding spectrum. The PA spectrum is a reflection of the rovibrational structure of excited molecules. In this case lines are assigned to the Hund's case (c) $0_{u}^{+}$or $1_{u}$ symmetry.

Details of the Born-Oppenheimer potential at short interatomic separation are insufficiently known for a quantitative 
prediction of the bound-state locations. Therefore, we have modified the short range of the ${ }^{3} \Pi_{u}$ potential to demonstrate how a spectrum can change. The $0_{u}^{+}$and $1_{u}$ potentials correlate to the ${ }^{3} \Pi_{u}$ and ${ }^{3} \Sigma_{u}^{+}$potentials at short range, respectively. Consequently, by changing the ${ }^{3} \Pi_{u}$ potential we can change the location of the $0_{u}^{+}$bound states while leaving the position of $1_{u}$ bound states virtually unchanged. It is convenient to define "bins" of the $0_{u}^{+}$potential as energy intervals with edges marked by the $0_{u}^{+}$energy levels calculated with a ${ }^{3} \Pi_{u}$ potential, such that the last bound state is exactly on the ${ }^{3} P_{1}+{ }^{1} S_{0}$ threshold. As the short-range ${ }^{3} \Pi_{u}$ potential is changed, there is always exactly one $0_{u}^{+}$bound state in each bin. The "bin edges" for $0_{u}^{+}$states are shown in Fig. [4

Figure 5 shows PA spectra for two ${ }^{3} \Pi_{u}$ potentials in order to illustrate the limiting cases of overlapping and nonoverlapping $0_{u}^{+}$and $1_{u}$ bands. The PA spectra are calculated using the lineshape formula derived in the previous section and take into account Doppler broadening. In both spectra the last five vibrational levels of $0_{u}^{+}$and $1_{u}$ symmetry are shown. The PA rate coefficient increases by nine orders of magnitude near each vibrational level. Observable trap loss is on the order of the $10^{-12} \mathrm{~cm}^{3} \mathrm{~s}^{-1}$. In principle, ion detection allows measurement of weaker PA lines than is possible with trap loss. On the frequency scale of the figure the location of the $1_{u}$ vibrational lines for the two potentials is almost the same.

A spectrum in which lines with $0_{u}^{+}$and $1_{u}$ symmetry are far apart from one another is shown in Fig. 5 5 a). In this case the projection $\Omega_{e}$ of the electronic angular momentum $j_{e}$ on the intermolecular axis is a good quantum number and bound states can be labelled by the Hund's case (c) coupling scheme. This kind of spectrum has been observed in preliminary experiments on Strontium near the ${ }^{1} S_{0}-{ }^{3} P_{1}$ line by Ido and Katori [50].

The rather small anisotropy of the long-range dispersion interaction of the $0_{u}^{+}$and $1_{u}$ potentials $\left(C_{6}\left(0_{u}^{+}\right) \approx C_{6}\left(1_{u}\right)\right.$ to within 5\%) can lead to a near coincidence of $0_{u}^{+}$and $1_{u}$ levels over a range of $v$. Such a case is shown in Fig. [5]b). For closely spaced doublets the projection $\Omega_{e}$ is not a good quantum number, and bound states should rather be labelled by the rotational angular momentum $l_{e}$ as in the Hund's case (e) coupling scheme. It can be clearly seen that in each doublet there is a strong and weak line corresponding to $l_{e}=0$ and $l_{e}=2$, respectively.

We have also studied the change in coupling scheme from Hund's case (c) to $e$ for bound states very close to the molecular thresholds. By changing the shape of the ${ }^{3} \Pi_{u}$ potential, the last bound state, initially attributed to $0_{u}^{+}$symmetry, smoothly approaches threshold. Simultaneously, the wave function smoothly changes its character as well. For a binding energy $\Delta_{e} / h=-0.026 \mathrm{GHz} 90 \%$ of the wave function has $\Omega_{e}=0$ character, while for $\Delta_{e} / h=-0.003 \mathrm{GHz} 90 \%$ of the wave function can be attributed to $l_{e}=0$. In other words, Hund's case (c) holds for binding energies larger than 0.026 $\mathrm{GHz}$.

In Fig. [5 and in the remainder of this paper the natural linewidth $\Gamma_{e, \text { nat }}$ is approximated by $2 \Gamma_{A}\left({ }^{3} P_{1}\right)=0.663 \mathrm{kHz}$. The natural linewidths calculated from the theory described in the appendices shows that the width is less than about four
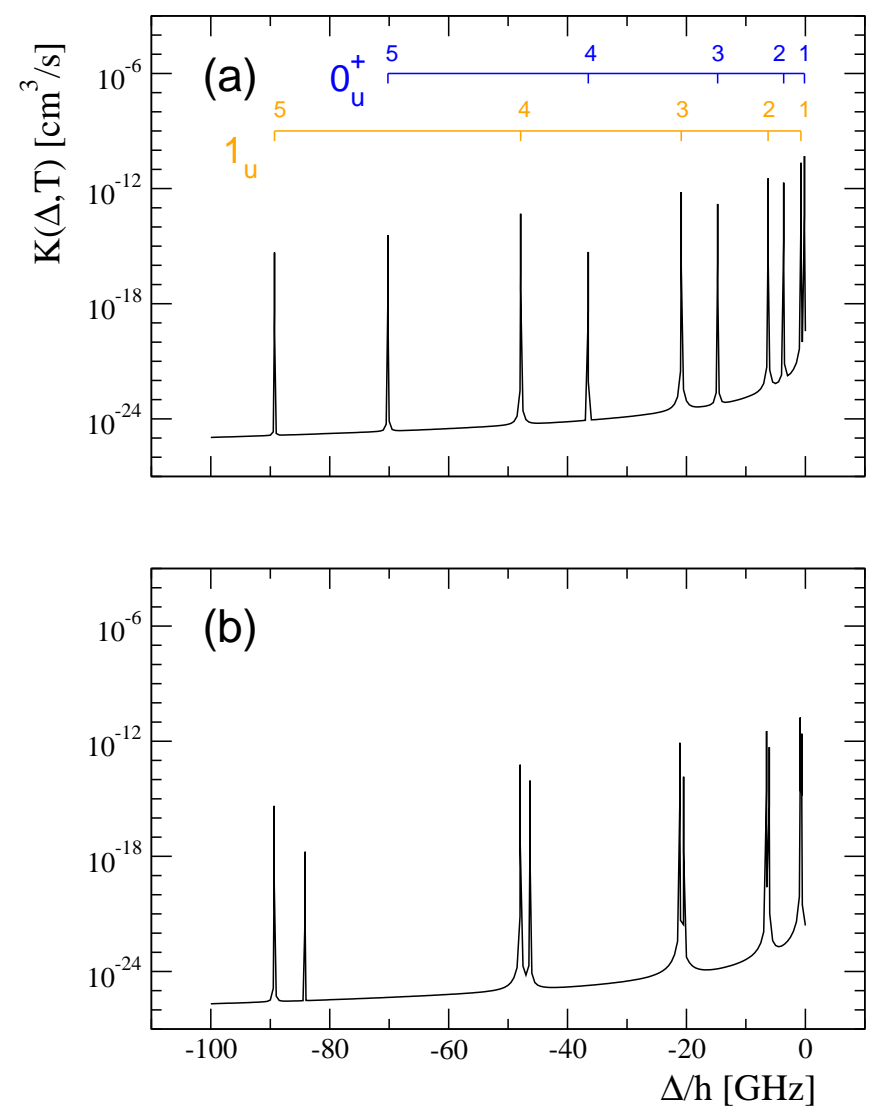

FIG. 5: Photoassociation spectra of calcium near the intercombination line for two ${ }^{3} \Pi_{u}$ potentials. Panel (a) is for the potential used in Fig. 4 Panel (b) is for a potential where the $0_{u}^{+}$and $1_{u}$ bands nearly overlap. The laser intensity is $1 \mathrm{~W} / \mathrm{cm}^{2}$ and the temperature of the gas is $T=1 \mu \mathrm{K}$. The $0_{u}^{+}$and $1_{u}$ vibrational assignment is shown in Panel A. The last vibrational level is labeled by " 1 ".

times $\Gamma_{A}\left({ }^{3} P_{1}\right)$ for the detunings shown in Fig. 5 We believe that our simple model can not quantitatively describe the natural widths. The treatment of the coupling between ${ }^{1} P$ and ${ }^{3} P$ states is not sufficiently accurate. For simplicity we assume the same natural linewidth for all lines.

Figure 6 displays the collision-energy dependence of the stimulated width $\Gamma_{e g}\left(\varepsilon_{r}\right)$ for the $0_{u}^{+}$lines shown in Fig. 5 a). The ground-state potential has a scattering length of $389.8 a_{0}$ and the laser intensity is $1 \mathrm{~W} / \mathrm{cm}^{2}$. The width rapidly increases for bound states closer to the threshold as the overlap of the bound and scattering wavefunction grows. Moreover, for collision energies smaller than $\varepsilon_{r} / k_{B}=100 \mu \mathrm{K}$ the width of all lines is proportional to $\sqrt{\varepsilon_{r}}$, satisfying the Wigner threshold law. For a $1 \mathrm{~W} / \mathrm{cm}^{2}$ laser intensity and collision energies less than $1 \mu \mathrm{K}$ the stimulated width for four of the vibrational levels is smaller than the natural width. Therefore, the lines are unsaturated for most of lines shown in Fig.5 The exception are those lines closest to resonance.

In order to show the temperature dependence of the shape of a line we have chosen line 2 of the $0_{u}^{+}$band in Fig. 5 a). Figure 7 shows the lineshape for a temperature of $1 \mathrm{mK}, 1$ $\mu \mathrm{K}$, and $1 \mathrm{nK}$ with and without Doppler broadening. In the 


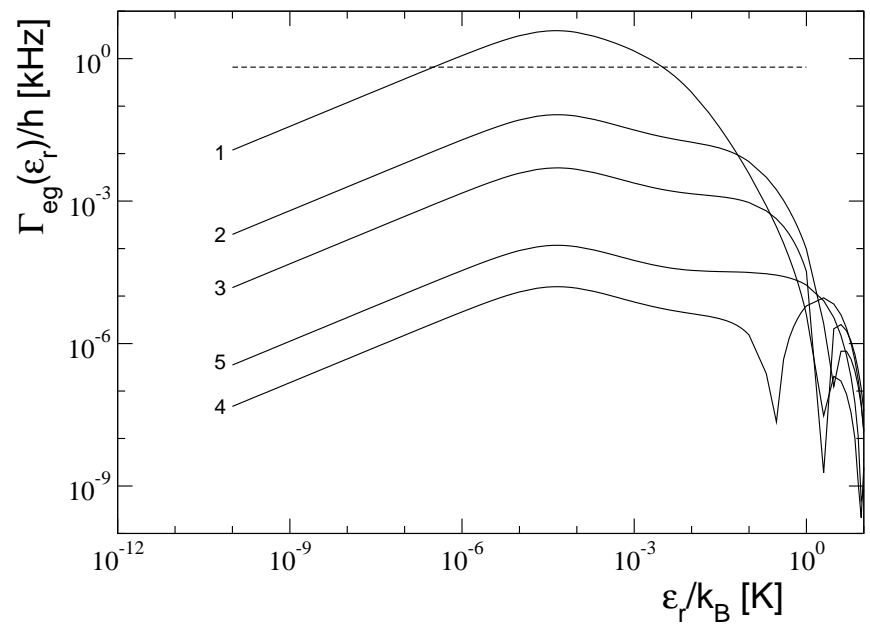

FIG. 6: The stimulated width as a function of the kinetic energy of a ${ }^{1} S+{ }^{1} S$ collision calculated for the five lines of the $0_{u}^{+}$band shown in panel (a) of Fig. 5 The laser intensity is $1 \mathrm{~W} / \mathrm{cm}^{2}$. The natural width $\Gamma_{e, \text { nat }} / h=0.663 \mathrm{kHz}$ is marked by the dashed line.
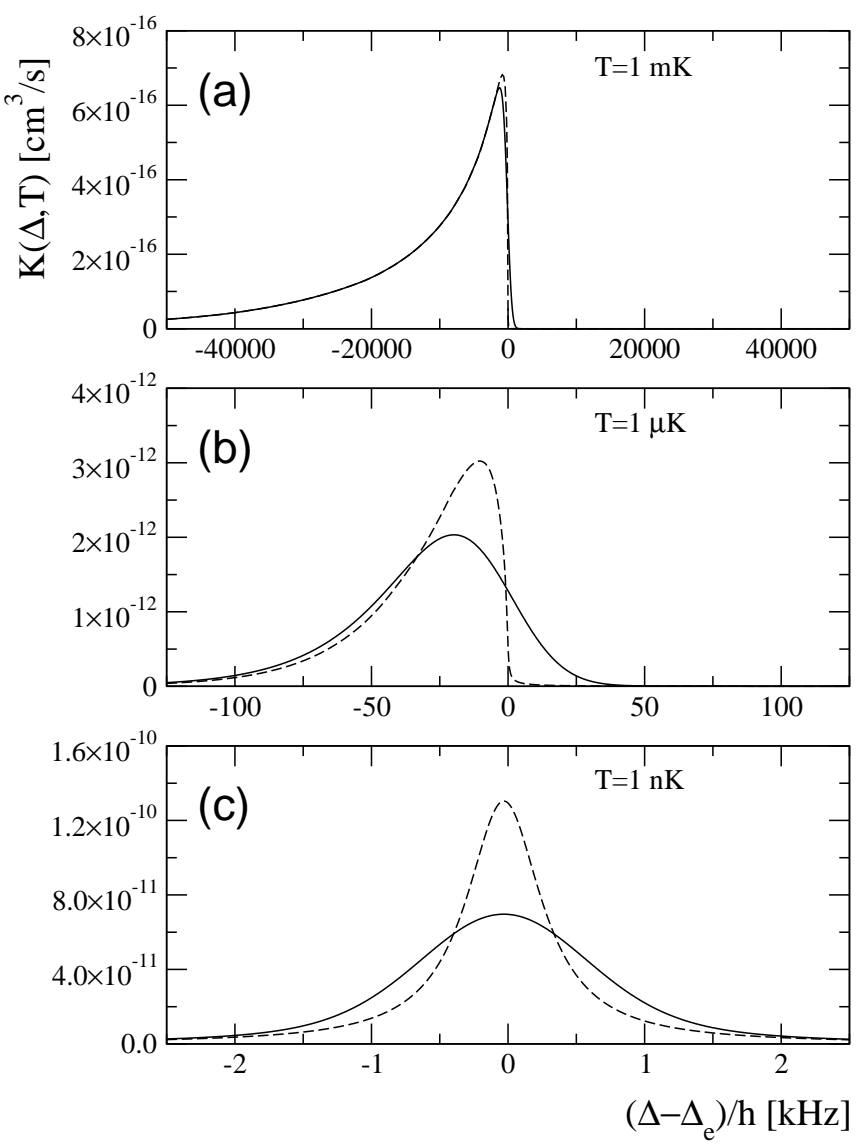

FIG. 7: The lineshape of the $0_{u}^{+}$line labelled 2 shown in panel (a) of Fig. 5 as a function of laser detuning. Panels (a), (b) and (c) show lineshapes at a temperature of $1 \mathrm{mK}, 1 \mu \mathrm{K}$ and $1 \mathrm{nK}$, respectively. Profiles that include and do not include Doppler broadening are marked by solid and dashed lines, respectively. The laser intensity is $1 \mathrm{~W} / \mathrm{cm}^{2}$. The quantity $\Delta_{e}$ is the binding energy of the $0_{u}^{+}$ rovibrational level relative to the ${ }^{1} S_{0}+{ }^{1} P_{1}$ dissociation limit. absence of Doppler broadening $\Delta_{D}=0$ in Eq. 6 For these three temperatures the thermal width $\Delta_{T} / h=20837 \mathrm{kHz}$, $20.8 \mathrm{kHz}$, and $0.021 \mathrm{kHz}$ and the Doppler width $\Delta_{D} / h=$ $694 \mathrm{kHz}, 21.9 \mathrm{kHz}$, and $0.694 \mathrm{kHz}$, respectively.

At a temperature of $1 \mathrm{mK}$ the line in Fig.7 a) has the typical "cut-off exponential" shape determined by thermal broadening and is only slightly affected by Doppler broadening. The width of line is on the order of ten $\mathrm{MHz}$ and the peak rate coefficient is $10^{-15} \mathrm{~cm}^{3} \mathrm{~s}^{-1}$. Such a low rate coefficient makes trap loss hard to detect in typical ultra-cold-atom experiments in a magneto-optical trap. In this case ion detection might be a sensitive alternative.

A $1 \mu \mathrm{K}$ atomic-gas temperature is close to the recoil temperature $T_{R}=1.11 \mu \mathrm{K}$. Under such conditions both thermal and Doppler broadening in the PA lineshape are comparable. Figure 7 (b) demonstrates a significant difference between a Doppler broadened profile and one without Doppler broadening. The width of the line is on the order of hundred $\mathrm{kHz}$ and the peak rate coefficient is $10^{-12} \mathrm{~cm}^{3} \mathrm{~s}^{-1}$. A trap-loss signal should be observable for such rate coefficients.

In Fig. 7 (c) a PA lineshape for a thermal gas at $1 \mathrm{nK}$ is shown. Typically for such low temperatures and sufficiently high densities an atomic gas could be Bose condensed(BEC) and a Boltzman distribution of atomic momentum should not be used. Here, we assume a low enough density that condensation has not occurred. The lineshape is an ordinary Voigt profile, which is determined by Doppler and natural broadening. The natural width $\Gamma_{e, \text { nat }}$ is $0.663 \mathrm{kHz}$. The line is Lorentzian if Doppler broadening is neglected. The width of the line is on the order of $\mathrm{kHz}$ and the peak rate coefficient is $10^{-10} \mathrm{~cm}^{3} \mathrm{~s}^{-1}$. Moreover, the molecular recoil energy $E_{\text {rec,mol }} / h=5.775 \mathrm{kHz}$ is significantly bigger than the width of the line.

The width of the lines in Fig. 7 varies by four orders of magnitude. The peak rate coefficient changes by five orders of magnitude. Clearly, for temperatures on the order of $1 \mu \mathrm{K}$ and below, photoassociation spectra should be observable. Moreover, Doppler broadening is an important factor and affects the shape of the lines significantly.

We have also analyzed the stimulated width of the $0_{u}^{+}$lines as a function of the scattering length in the ${ }^{1} S_{0}+{ }^{1} S_{0}$ ground state and the binding energy $\Delta_{e}$ of the excited $0_{u}^{+}$bound states. The scattering length is varied within the range allowed by experiment [27]. The scattering length is varied by slight modifications of the short-range part of the ground-state potential. The binding energy of the $0_{u}^{+}$bound states are changed by modifying the ${ }^{3} \Pi_{u}$ potential as discussed above.

Figure 8 shows the stimulated width at a collision energy $\varepsilon_{r} / k_{B}=1 \mu \mathrm{K}$ as a function of $a_{\text {scat }}$ and $\Delta_{e}$. The figure shows multiple nearly vertical dark structures, where the width is nearly zero. There are two kinds of these structures: ones accompanied by a parallel bright feature, where $\Gamma_{e g}$ is large, and those without. The latter structures are not quite vertical for smaller $a_{\text {scat }}$. The "first kind" of structure occurs when a $0_{u}^{+}$bound state coincides with a $1_{u}$ bound state. This mixing is independent of any ground state scattering property and therefore the structures are vertical. The "second kind" of dark structures occur when the overlap of the scattering wave- 


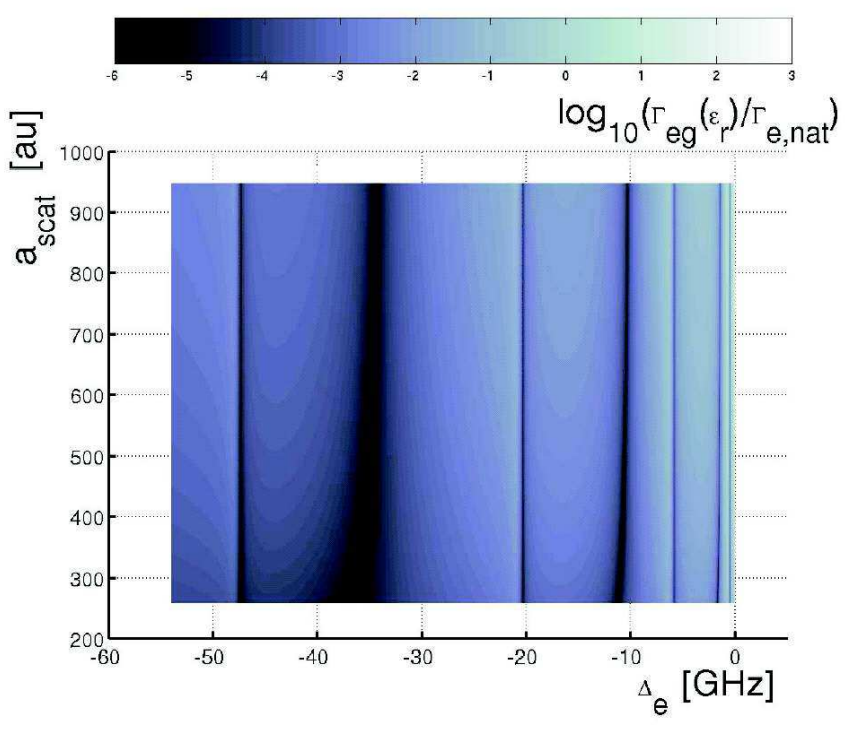

FIG. 8: The stimulated line width of the $0_{u}^{+}$band as a function of their line position (binding energy) $\Delta_{e}$ and scattering length $a_{\text {scat }}$ of the ground state potential. The laser intensity is $1 \mathrm{~W} / \mathrm{cm}^{2}$ and the kinetic energy of the collision is $\varepsilon_{r} / k_{B}=1 \mu \mathrm{K}$.

function and the excited bound state vanishes. The shape of the scattering wavefunction near the outer turning point of the excited bound state does not change much when the scattering length is on the order of a few hundred $a_{0}$. Therefore, these structures are nearly vertical. Near $a_{\text {scat }}=300 a_{0}$ a departure from vertical can be observed.

For photoassociation near strongly-allowed atomic transitions, such as occur in alkali-metal experiments 15, 18] and near the ${ }^{1} P_{1}$ line of the alkaline earths[19], the overlap vanishes for excited bound states with outer turning points near the nodes of the ground-state wave function. Their detuning $\Delta_{e}$ can be found with the help of the reflection approximation [14, 28], which says that the Franck-Condon factor is proportional to the square of the ground state wavefunction at the position $r_{C}$ for which the difference in the excited and ground state potentials equals the photon frequency.

In our case the reflection approximation cannot be applied because the asymptotic potentials in the ground and excited state are similar. The ground-state wave function has nodes at $26.5 \mathrm{au}, 32.4 \mathrm{au}$, and $46.1 \mathrm{au}$ for a scattering length $a_{\text {scat }}=389.8 \mathrm{au}$ and a collision energy $\varepsilon_{r} / k_{B}=1 \mu \mathrm{K}$. For the reflection approximation the relation between detuning and outer turning point of the excited bound states is determined from a potential that is the sum of the Hund's case (c) potential $V\left(0_{u}^{+}\right)$and the rotational correction $\left[2+J_{e}\left(J_{e}+\right.\right.$ 1)] $\hbar^{2} /\left(2 \mu r^{2}\right)$. The potential $V\left(0_{u}^{+}\right)$approaches the ${ }^{3} \Pi_{u}$ potential at large internuclear separation and $J_{e}=1$. These ground state nodes correspond to outer turning points detunings of $-51.3 \mathrm{GHz},-15.0 \mathrm{GHz}$, and $-1.6 \mathrm{GHz}$, respectively. These detunings do not correspond with dark lines in Fig. 8

Appendix [C gives another perspective of Fig. 8 The data are described in terms of a near threshold vibrational quantum number instead of the binding energy. Integer values of this

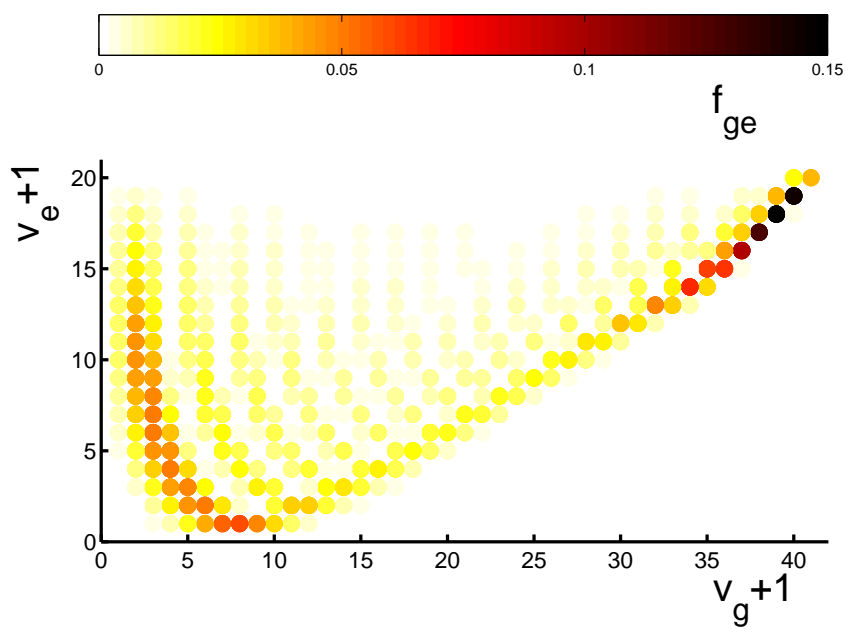

FIG. 9: The fraction $f_{g e}$ of molecules in rovibrational state $v_{g}, J_{g}=$ 0 of the ${ }^{1} \Sigma_{g}^{+}$potential produced by natural decay of rovibrational state $v_{e}, J_{e}=1$ of $0_{u}^{+}$symmetry.

quantum number are related to the bins defined in Fig. 4 This discussion is not crucial for the main thrust of the paper and, therefore, has been placed in an appendix. It, however, gives a deeper understanding of the physics involved and is worth presenting.

Finally, we have investigated the possibility of creating cold molecules in the ground electronic state via the photoassociation process. The similar $C_{6} / r^{6}$ dependence of the ground and excited potentials can make such a process more efficient than making ground state molecules through excited states in which the asymptotic form of the potential is $C_{3} / r^{3}$. We have calculated rates for bound-bound transitions between $0_{u}^{+}$excited states and ${ }^{1} \Sigma_{g}^{+}$ground states. From these rates we have calculated the fraction of ground-state molecules, which after photoassociation are formed by natural decay. Figure 9 presents the fraction $f_{g e}$ of molecules in rovibrational state $v_{g}, J_{g}=0$ of the ${ }^{1} \Sigma_{g}^{+}$potential produced by natural decay of rovibrational state $v_{e}, J_{e}=1$ of $0_{u}^{+}$symmetry. The fraction of a $v_{g}, J_{g}=0$ molecule is no more than $15 \%$. For $v_{e}>10$ high $v_{g}$ vibrational levels of the ground state potential are produced, while for $v_{e}<10$ low $v_{g}$ levels are produced. Nevertheless, even for high $v_{e}$ a few percent of the ground-state molecules have low $v_{g}$. The fraction of molecules decaying to $J_{g}=2$ vibrational states of the ${ }^{1} \Sigma_{g}$ potential have also been calculated, but are not shown. It was found that the fraction of excited molecules decaying to the $J_{g}=2$ vibrational states is bigger than those decaying to the $J_{g}=0$ vibrational states. Rotational states with $J_{g}=1$ do not exist because of Bose symmetrization (See appendix $\mathrm{A}$.

Adding the $J_{g}=0$ and 2 fractions $f_{g e}$ of molecules shows that most of the spontaneous emission of a $v_{e}, J_{e} 0_{u}^{+}$rovibrational level goes to ground molecular bound states. In fact, for $v_{e}<19$ more than $80 \%$ of the decay is to molecular states. The only exception is the last vibrational level, $v_{e}=19$, corresponding to line $1 \mathrm{in} \mathrm{Fig.5} 5$ a), for which $30 \%$ of the decay is to molecular states. We have also found that the spontaneous 
decay rate of deeply-bound excited states can be one order of magnitude larger than $2 \Gamma_{A}\left({ }^{3} P_{1}\right) / \hbar$.

\section{CONCLUSIONS}

We have developed a description for the shape of photoassociation lines for weak transitions in which the natural width is smaller than the Doppler width. The lineshape theory includes Doppler broadening and a photon recoil shift. It was shown that the Doppler effect significantly affects the PA lineshape when the gas temperature is on the order of the recoil temperature and below.

A model calculation for calcium has been carried out. It is an example of photoassociation near the intercombination line of alkaline-earth atoms. We find that photoassociation spectroscopy should be possible at calcium gas temperatures on the order of $\mu \mathrm{K}$ and below. In addition, it was shown that when the long-range potential of the ground and excited state are similar, the reflection approximation [10, 14, 28] incorrectly estimates the stimulated width and strength of PA lines. Finally, we have indicated that excited molecules are very likely to decay to vibrational levels of the electronic ground state. This can be used as an effective way to produce cold molecules.

\section{Acknowledgments}

The authors wish to express their gratitude to A. Derevianko for making his calculations of dispersion coefficients available before publication. This work has been partially supported by the U.S. Office of Naval Research. The research is part of the program of the National Laboratory FAMO in Torun, Poland.

\section{APPENDIX A: CLOSE-COUPLING CALCULATIONS}

The quantum theory of slow-atom collisions [51, 52] allows a quantitative description of the scattering process and bound states. We apply this theory to describe the slow collisions and bound states of alkaline-earth atoms.

Scattering or bound states of two identical alkaline-earth atoms without nuclear spin can be described in the basis

$$
\left|S L j l J M_{J} ; p\right\rangle \equiv \sum_{m_{j}, m_{l}}\left\langle j l m_{j} m_{l} \mid J M_{J}\right\rangle\left|S L j m_{j} ; \sigma\right\rangle Y_{l m_{l}}(\theta, \phi),
$$

where the $\left|S L j m_{j} ; \sigma\right\rangle$ describe the electronic state of the molecule and $Y_{l m}(\theta, \phi)$ is a spherical harmonic describing the nuclear rotation. The quantity $\vec{L}$ is the total electron orbital angular momentum, $\vec{S}$ is the total electron spin angular momentum, $\vec{\jmath}=\vec{L}+\vec{S}$ is the total electron angular momentum, $\vec{l}$ is the rotational angular momentum, and $\vec{J}=\vec{l}+\vec{\jmath}$ is the total angular momentum. The projections of $\vec{\jmath}, \vec{l}$, and $\vec{J}$ on a space-fixed $z$-axis are $m_{j}, m_{l}$, and $M_{J}$, respectively. The quantity $p$ is the total parity. Gerade $(g, \sigma=+1)$ and ungerade $(u, \sigma=-1)$ electronic states correspond to total parity $p=+1$ and $p=-1$ states, respectively. This is a consequence of the more general rule for atoms with nonzero nuclear spin $p=\sigma(-1)^{2 I}$, where $I$ is the atomic nuclear spin. The total parity restricts the allowed $l$ by $p=p_{A} p_{B}(-1)^{l}$, where $p_{A}$ and $p_{B}$ are the atomic parities. The atomic parity is +1 for the ground ${ }^{1} S$ state and -1 for excited ${ }^{1} P$ and ${ }^{3} P$ states.

The molecular Hamiltonian $H=T+H_{A}+V_{\text {int }}+$ $V_{\text {rot }}$ is calculated in the $\left|S L j l J M_{J} ; p\right\rangle$ basis. Here, $T=$ $-\left(\hbar^{2} /(2 \mu)\right) d^{2} / d r^{2}$ is the kinetic energy operator, $H_{A}$ is the atomic Hamiltonian, $V_{\text {int }}$ are the nonrelativistic BornOppenheimer potentials, and $V_{\text {rot }}=\hbar^{2} \vec{l}^{2} /\left(2 \mu r^{2}\right)$ describes the rotational energy. The matrix elements for the kinetic and rotational energy are diagonal in this basis. In fact, $\left\langle S L j l J M_{J} ; p|T| S L j l J M_{J} ; p\right\rangle=-\left(\hbar^{2} /(2 \mu)\right) d^{2} / d r^{2}$ and $\left\langle S L j l J M_{J} ; p\left|V_{\text {rot }}\right| S L j l J M_{J} ; p\right\rangle=\hbar^{2} l(l+1) /\left(2 \mu r^{2}\right)$.

The matrix elements for the Born-Oppenheimer potentials are calculated in two steps. The first step involves transforming the molecular electronic state $\left|S L j m_{j} ; \sigma\right\rangle$ into a bodyfixed coordinate system. That is into a superposition of $|S L j \Omega ; \sigma\rangle$ states, where $\Omega$ is the projection of $\vec{j}$ on the internuclear axis. After some algebra the matrix elements are given by

$$
\begin{aligned}
& \left\langle S^{\prime} L^{\prime} j^{\prime} l^{\prime} J^{\prime} M_{J}^{\prime} ; p^{\prime}\left|V_{\mathrm{int}}\right| S L j l J M_{J}, p\right\rangle= \\
& \delta_{p^{\prime}, p} \delta_{J^{\prime}, J} \delta_{M_{J}^{\prime}, M_{J}} \sqrt{\frac{\left(2 l^{\prime}+1\right)(2 l+1)}{(2 J+1)^{2}}} \\
& \sum_{\Omega}\left\langle j^{\prime} l^{\prime} \Omega 0 \mid J^{\prime} \Omega\right\rangle\langle j l \Omega 0 \mid J \Omega\rangle\left\langle S^{\prime} L^{\prime} j^{\prime} \Omega ; \sigma\left|V_{\mathrm{int}}\right| S L j \Omega ; \sigma\right\rangle,
\end{aligned}
$$

i.e. the operator $V_{\text {int }}$ is diagonal in $J, M_{J}$, and $p$ but not diagonal in $j$ and $l$. A similar transformation is discussed by Napolitano et al. [33] in the context of ultra-cold collisions between atoms in the ${ }^{1} S_{0}$ and ${ }^{1} P_{1}$ states.

The next step is to express the body-fixed electronic states $|S L j \Omega ; \sigma\rangle=\sum_{\Sigma, \Lambda}\langle S L \Sigma \Lambda \mid j \Omega\rangle|S L \Sigma \Lambda ; \sigma\rangle$ in terms of $|S L \Sigma \Lambda ; \sigma\rangle$, where $\Sigma$ and $\Lambda$ are projections of $S$ and $L$ along the internuclear axis. In other words,

$$
\begin{aligned}
& \left\langle S^{\prime} L^{\prime} j^{\prime} \Omega^{\prime} ; \sigma^{\prime}\left|V_{\mathrm{int}}\right| S L j \Omega ; \sigma\right\rangle= \\
& \sum_{\Sigma^{\prime}, \Lambda^{\prime}, \Sigma, \Lambda}\left\langle S^{\prime} L^{\prime} \Sigma^{\prime} \Lambda^{\prime} \mid j^{\prime} \Omega^{\prime}\right\rangle\langle S L \Sigma \Lambda \mid j \Omega\rangle \\
& \left\langle S^{\prime} L^{\prime} \Sigma^{\prime} \Lambda^{\prime} ; \sigma^{\prime}\left|V_{\mathrm{int}}\right| S L \Sigma \Lambda ; \sigma\right\rangle .
\end{aligned}
$$

The Born-Oppenheimer potentials are diagonal in this $|S L \Sigma \Lambda ; \sigma\rangle$ basis and the diagonal matrix elements are $\left\langle S L \Sigma \Lambda ; \sigma\left|V_{\text {int }}\right| S L \Sigma \Lambda ; \sigma\right\rangle=V_{\text {int }}\left({ }^{2 S+1}|\Lambda|_{\sigma}\right)$.

Finally, we calculate matrix elements of the Hamiltonian $H_{A}$ for two non-interacting atoms, where one atom is always in the ${ }^{1} \mathrm{~S}_{0}$ state while the other atom can be in the state ${ }^{1} \mathrm{~S}_{0}$, ${ }^{1} \mathrm{P}_{1}$, or ${ }^{3} \mathrm{P}_{2,1,0}$. Because one atom is in the ${ }^{1} \mathrm{~S}_{0}$ state, the molecular angular momenta $S, L$, and $j$ in Eq. A1 are equivalent to those of the second atom. 
TABLE I: The values of energies and lifetimes of $\mathrm{Ca}$ atomic states used in this paper.

\begin{tabular}{c|rr}
\hline \hline${ }^{2 S+1} L_{j}$ & $E_{A}\left({ }^{2 S+1} L_{j}\right)\left[\mathrm{cm}^{-1}\right]$ & $\tau_{A}\left({ }^{2 S+1} L_{j}\right)$ \\
\hline${ }^{1} P_{1}$ & 23652.304 & $4.59 \mathrm{~ns}$ \\
${ }^{3} P_{2}$ & 15315.943 & \\
${ }^{3} P_{1}$ & 15210.063 & $0.48 \mathrm{~ms}$ \\
${ }^{3} P_{0}$ & 15157.901 & \\
${ }^{1} S_{0}$ & 0.000 & \\
\hline \hline
\end{tabular}

A realistic description of the atomic Hamiltonian should include relativistic coupling between singlet ${ }^{1} P_{1}$ and triplet ${ }^{3} P_{1}$ states. Therefore, the atomic Hamiltonian is not diagonal in the basis of Eq. A1]. Following Mies et al.[39] "dressed" electronic states for $j=1$, which are a mixture of singlet and triplet, are introduced as follows

$$
\begin{aligned}
& \left|\widetilde{S}=0, \operatorname{Ljm}_{j} ; \sigma\right\rangle \equiv \\
& \quad \cos (\alpha)\left|S=0, \operatorname{Lj}_{j} ; \sigma\right\rangle+\sin (\alpha)\left|S=1, L j m_{j} ; \sigma\right\rangle
\end{aligned}
$$

and

$$
\begin{aligned}
\mid \widetilde{S} & \left.=1, \operatorname{Ljm}_{j} ; \sigma\right\rangle \equiv \\
& -\sin (\alpha)\left|S=0, \operatorname{Ljm}_{j} ; \sigma\right\rangle+\cos (\alpha)\left|S=1, L j m_{j} ; \sigma\right\rangle
\end{aligned}
$$

where $\alpha$ is a small mixing angle. For $j=0$ and 2 we have $\left|\widetilde{S} L j m_{j} ; \sigma\right\rangle=\left|S L j m_{j} ; \sigma\right\rangle$. We assume that in this dressed basis the atomic Hamiltonian is diagonal with diagonal matrix elements $\left\langle\widetilde{S} L j l J M_{J} ; p\left|H_{A}\right| \widetilde{S} L j l J M_{J} ; p\right\rangle=E_{A}\left({ }^{2 \widetilde{S}+1} L_{j}\right)$, where $E_{A}\left({ }^{2 \widetilde{S}+1} L_{j}\right)$ is the energy of the dressed state ${ }^{2 \widetilde{S}+1} L_{j}$ relative to the ${ }^{1} S_{0}$ ground state.

The mixing angle $\alpha$ is determined by the requirement that the dressed basis $\left|\widetilde{S} L j m_{j} ; \sigma\right\rangle$ reproduces the experimental transition probabilities between the excited ${ }^{1,3} \mathrm{P}_{1}$ states and the ground ${ }^{1} \mathrm{~S}_{0}$ state. The angle can then be related to the ratio of the experimental dipole moments of these transitions. In fact, we used $\tan (\alpha)=$ $\sqrt{\left[E_{A}\left({ }^{1} P_{1}\right) / E_{A}\left({ }^{3} P_{1}\right)\right]^{3}\left[\tau_{A}\left({ }^{1} P_{1}\right) / \tau_{A}\left({ }^{3} P_{1}\right)\right]}$. This approach is only an approximation of the description of a real alkalineearth atom[39]. For the purposes of this paper, however, it is sufficient. The values for the energies and lifetimes have been obtained from Refs. [19, 47, 53] and listed in Table 凹 The experimental results on the ${ }^{3} P_{1}$ lifetime are compared by Drozdowski et al. [53] and some uncertainty still exist. We have chosen the estimated value of Ref. [19], which lies between the experimental values.

The molecular Hamiltonian $H$ conserves $J, M_{J}$, and $p$. In fact, the matrix elements of $H$ in the basis of Eq. A1 for given $J$ and $p$ are independent of $M_{J}$. It is convenient to introduce the channels $|\gamma\rangle=\left|S L j l J M_{J} ; p\right\rangle$, $|\widetilde{\gamma}\rangle=\left|\widetilde{S} L j l J M_{J} ; p\right\rangle$, and note that $\left\langle\widetilde{\gamma}^{\prime}|H| \widetilde{\gamma}\right\rangle=$ $\sum_{\gamma^{\prime}, \gamma}\left\langle\widetilde{\gamma}^{\prime} \mid \gamma^{\prime}\right\rangle\left\langle\gamma^{\prime}|H| \gamma\right\rangle\langle\gamma \mid \widetilde{\gamma}\rangle$. Close-coupling equations for the molecular wave function $|\Psi\rangle=\sum_{\widetilde{\gamma}}|\widetilde{\gamma}\rangle F_{\widetilde{\gamma}}(r) / r$ can be
TABLE II: The allowed $J_{e}=1, p_{e}=-1$ and $M_{e}=0, \pm 1$ dressed channels of interacting ground- and excited-state alkalineearth atoms.

\begin{tabular}{c|cccc}
\hline \hline$\left.\widetilde{\gamma}_{e}\right\rangle$ & $\widetilde{S}_{e}$ & $L_{e}$ & $j_{e}$ & $l_{e}$ \\
\hline 1 & 1 & 1 & 1 & 0 \\
2 & 1 & 1 & 1 & 2 \\
3 & 1 & 1 & 2 & 2 \\
4 & 0 & 1 & 1 & 0 \\
5 & 0 & 1 & 1 & 2 \\
\hline \hline
\end{tabular}

written as

$$
-\frac{\hbar^{2}}{2 \mu} \frac{d^{2}}{d r^{2}} F_{\widetilde{\gamma}}(r)+\sum_{\widetilde{\gamma}^{\prime}}\left\langle\widetilde{\gamma}\left|H_{A}+V_{\text {int }}+V_{\text {rot }}\right| \widetilde{\gamma}^{\prime}\right\rangle F_{\widetilde{\gamma}^{\prime}}(r)=E F_{\widetilde{\gamma}}(r),
$$

where $E$ is the total molecular energy. These coupled Schrödinger equations are solved numerically to find scattering and bound states.

The collision between two ground state atoms can be solved separately from that of a ground plus an excited state atom. Moreover, for photoassociation of $\mathrm{Ca}$ we need scattering solutions of the Schrödinger equation for ground state collisions and bound states for ground-excited molecules.

For the collision of two ground-state atoms ${ }^{1} \mathrm{~S}_{0}+{ }^{1} \mathrm{~S}_{0}$ there is only a single channel for given $J_{g}, M_{g}$ and $p_{g}$. The channel is $\left|\gamma_{g}\right\rangle=\left|S_{g} L_{g} j_{g} l_{g} J_{g} M_{g} ; p_{g}\right\rangle$ with $S_{g}=0, L_{g}=0$, $j_{g}=0$ and $l_{g}=J_{g}$. The two ${ }^{40} \mathrm{Ca}$ atoms are indistinguishable bosons and, therefore, the wavefunction must be symmetric under exchange of atoms and only channels with positive parity $p=+1$ exist. Consequently, only even $l_{g}=0,2,4, \ldots$ are allowed and the atoms interact on the gerade electronic state ${ }^{1} \Sigma_{g}^{+}$. Solving the Schrödinger equation with $E=\varepsilon_{r}>0$ we obtain the scattering wavefunction $\left|\Psi_{g}^{+}\left(\varepsilon_{r}, J_{g}, M_{g}\right)\right\rangle=$ $\left|\gamma_{g}\right\rangle F_{\gamma_{g}}^{+}(r) / r$. This wavefunction occurs in the stimulated width defined by Eq. (5). For large $r$ the wavefunction goes to $F_{\gamma_{a}}^{+}(r) \rightarrow \sqrt{2 \mu /\left(\pi \hbar^{2} k_{r}\right)} \sin \left(k_{r} r+\pi l_{g} / 2+\eta_{l_{g}}\right) \exp \left(i \eta_{l_{g}}\right)$ [14, 15]. Our interest will be in the solution for $J_{g}=l_{g}=0$ or $s$-wave collisions. For collisions at ultralow temperatures other partial waves do not contribute significantly.

For molecules formed by a ground- and excited-state atom there are multiple channels involved. The number of channels is determined by $J_{e}$ and $p_{e}$. Photon selection rules limit the allowed total angular momentum of the excited bound states to $J_{e}=J_{g}, J_{g} \pm 1$ and their parity to $p_{e}=-p_{g}$. For $J_{g}=0$ or $s$ wave collisions PA can only make $J_{e}=1$ and $p_{e}=-1$ bound states. Table Ilists the five channels for $J_{e}=1$ and $p_{e}=-1$. The first two channels correspond to ${ }^{1} \mathrm{~S}_{0}+{ }^{3} \mathrm{P}_{1}$ states. There are no channels with $j_{e}=0$ and, since the atomic energies satisfy $E_{A}\left({ }^{3} P_{0}\right)<E_{A}\left({ }^{3} P_{1}\right)<E_{A}\left({ }^{3} P_{2}\right)$, predissociation of bound states below the ${ }^{1} S_{0}+{ }^{3} P_{1}$ limit does not occur. In fact, this is true for all odd $J_{e}$ and $p_{e}=-1$.

The numerical solutions of the Schrödinger equation for the scattering collision in the ground state are calculated using the Numerov method [54, 55] implemented in the close 
coupling code developed by Mies, Julienne and Sando [56]. The coupled-channel bound states calculations for the excited state are carried out using the discrete variable representation (DVR) [57, 58] following Tiesinga et al. [59].

\section{APPENDIX B: INTERACTION WITH LIGHT}

In our treatment of the photoassociation process we need the stimulated width, defined by Eq. (4), between the scattering ground state and the excited bound states. The stimulated width can be expressed as

$$
\Gamma_{e g}\left(\varepsilon_{r}\right)=2 \pi\left|\sum_{\widetilde{\gamma}_{e}} \int_{0}^{\infty} d r\left\langle\widetilde{\gamma}_{e}\left|V_{\text {las }}\right| \gamma_{g}\right\rangle F_{\widetilde{\gamma}_{e}}^{*}(r) F_{\gamma_{g}}^{+}(r)\right|^{2}
$$

where the wavefunctions are expressed in terms of channel functions $F_{\widetilde{\gamma}_{e}}(r)$ and $F_{\gamma_{e}}^{+}(r)$ and $\left\langle\widetilde{\gamma}_{e}\left|V_{\text {las }}\right| \gamma_{g}\right\rangle$ are matrix elements between channels in the ground and excited state. If we only consider dipole transitions and neglect retardation effects, this matrix element is independent of the interatomic separation $r$ and given by

$$
\left\langle\widetilde{\gamma}_{e}\left|V_{\text {las }}\right| \gamma_{g}\right\rangle=\sqrt{\frac{2 \pi I}{c}} \frac{1}{\sqrt{4 \pi \epsilon_{0}}}\left\langle\widetilde{\gamma}_{e}\left|\vec{d} \cdot \vec{e}_{q}\right| \gamma_{g}\right\rangle
$$

where the laser has intensity $I$ and polarization $\vec{e}_{q}, \epsilon_{0}$ the permittivity of vacuum, and $\left\langle\widetilde{\gamma}_{e}\left|\vec{d} \cdot \vec{e}_{q}\right| \gamma_{g}\right\rangle=\sum_{\gamma_{e}}\left\langle\widetilde{\gamma}_{e} \mid \gamma_{e}\right\rangle\left\langle\gamma_{e}\right| \vec{d}$. $\vec{e}_{q}\left|\gamma_{g}\right\rangle$ is the molecular dipole matrix element between spin channels.

Napolitano et al [33] discuss the connection between molecular and atomic dipole matrix elements. A similar approach is used here to find matrix elements. The molecular dipole matrix elements between ground and excited state are

$$
\begin{aligned}
& \left\langle S_{e} L_{e} j_{e} l_{e} J_{e} M_{e} ; p_{e}\left|d_{q}^{K}\right| S_{g} L_{g} j_{g} l_{g} J_{g} M_{g} ; p_{g}\right\rangle= \\
& \quad \sqrt{\frac{\left(2 l_{e}+1\right)\left(2 l_{g}+1\right)}{\left(2 J_{e}+1\right)^{2}}}\left\langle J_{g} K M_{g} q \mid J_{e} M_{e}\right\rangle \\
& \sum_{\Omega_{g}, \Omega_{e}, q^{\prime}}\left\langle J_{g} K \Omega_{g} q^{\prime} \mid J_{e} \Omega_{e}\right\rangle\left\langle j_{e} l_{e} \Omega_{e} 0 \mid J_{e} \Omega_{e}\right\rangle\left\langle j_{g} l_{g} \Omega_{g} 0 \mid J_{g} \Omega_{g}\right\rangle \\
& \left\langle S_{e} L_{e} j_{e} \Omega_{e} ; \sigma_{e}\left|d_{q^{\prime}}^{K}\right| S_{g} L_{g} j_{g} \Omega_{g} ; \sigma_{g}\right\rangle
\end{aligned}
$$

where the spherical tensor operator $d_{q}^{K}=\vec{d} \cdot \vec{e}_{q}$ with $K=1$ and $q=-1,0$, or +1 . In Eq. B3 we have expressed the dipole operator in a body-fixed coordinate system. For both singlet $S=0$ ground and excited states we realize that $|S L j \Omega ; \sigma\rangle=|S L \Sigma \Lambda ; \sigma\rangle$ and thus $j=L$ and $\Omega=\Lambda$. It then follows

$$
\begin{gathered}
\left\langle S_{e} L_{e} j_{e} \Omega_{e} ; \sigma_{e}\left|d_{q}^{K}\right| S_{g} L_{g} j_{g} \Omega_{g} ; \sigma_{g}\right\rangle= \\
\left\langle S_{e} L_{e} \Sigma_{e} \Lambda_{e} ; \sigma_{e}\left|d_{q}^{K}\right| S_{g} L_{g} \Sigma_{g} \Lambda_{g} ; \sigma_{g}\right\rangle,
\end{gathered}
$$

for electronic states with zero total electron $\operatorname{spin} S_{e}=S_{g}=0$. In the $|S L \Sigma \Lambda ; \sigma\rangle$ basis the dipole operator can be evaluated in terms of the atomic linewidth, $\Gamma_{A}\left({ }^{1} P_{1}\right)$, of the ${ }^{1} P_{1}$ atomic state decaying to the ground ${ }^{1} S_{0}$ state. There are four distinct matrix elements $\left\langle 010 \Lambda ; \sigma\left|d_{\Lambda}^{1}\right| 0000 ; g\right\rangle=d\left({ }^{1}|\Lambda|_{\sigma}\right)$ given by

$$
d\left({ }^{1}|\Lambda|_{\sigma}\right)=\sqrt{\frac{3}{4}\left(\frac{\lambda_{A}\left({ }^{1} P_{1}\right)}{2 \pi}\right)^{3} 4 \pi \epsilon_{0} \Gamma_{M}\left({ }^{1}|\Lambda|_{\sigma}\right)},
$$

where $\Gamma_{M}\left({ }^{1}|\Lambda|_{\sigma}\right)$ is the molecular linewidth. The $d\left({ }^{1}|\Lambda|_{\sigma}\right)$ are $r$-independent quantities as we neglect retardation [19, 48, 49] and, therefore, the molecular linewidth can be well approximated by $\Gamma_{M}\left({ }^{1}|\Lambda|_{u}\right)=2 \Gamma_{A}\left({ }^{1} P_{1}\right)$ for ungerade states and $\Gamma_{M}\left({ }^{1}|\Lambda|_{g}\right)=0$ for gerade states. In our phase convention the $d\left({ }^{1}|\Lambda|_{\sigma}\right)$ are positive. The molecular dipole matrix elements between the triplet $S_{e}=1$ and the ground $S_{g}=0$ states are zero.

\section{APPENDIX C: NEAR THRESHOLD VIBRATIONAL QUANTUM NUMBER}

Following LeRoy and Bernstein [60] the JWKB quantum condition for the eigenvalues of energies $E$ for a potential $V(r)$ is that $\Phi(E)=v \pi$ where $v$ is an integer and the phase $\Phi(E)=\Phi_{J W K B}(E)-\pi / 2$. Here $\Phi_{J W K B}(E)=\int_{r_{1}}^{r_{2}} d r \kappa(E, r)$ is the semiclassical phase integral of $\kappa(E, r)=\sqrt{(E-V(r)) 2 \mu / \hbar}$ calculated between the classical turning points $r_{1}$ and $r_{2}$. This criterion is valid for deeply bound states but breaks down near the dissociation limit[61]. It can be shown that for energies $E$ close to the threshold this condition still holds [62] if the phase $\Phi(E)$ is modified in the following way: $\Phi(E)=\Phi_{J W K B}(E)-$ $\pi / 2-\pi /(2 q-4)$ for long-range potentials of the form $V(r)=C_{q} / r^{q}$. A recent discussion on improvements to the LeRoy-Bernstein approach can be found in Ref. [63].

The phase $\Phi(E)$ allows us to define a generalized vibrational quantum number as $\left(\Phi(E)-v_{0} \pi\right) / \pi$, a continuous function of energy $E$, where $v_{0}$ is an arbitrary constant, which does not need to be an integer. For $v_{0}=0$ the generalized vibrational quantum number is $0,1, \ldots, v_{\max }-1$, or $v_{\max }$ when $E$ is equal to the energy of a bound state. Here, a zero value corresponds to the most deeply bound state and $v_{\max }$ is the vibrational quantum number of the last bound state. At the dissociation limit this generalized vibrational quantum number equals $v_{P}=\Phi(0) / \pi$ and, in general, can have a non-integer value 60].

In the analysis of bound states near the threshold it is convenient to set $v_{0}=v_{D}$ or $v_{0}=v_{\max }+1$. In the first case the generalized vibrational quantum number is equal to zero at threshold. Negative integers $-1,-2,-3, \ldots$ correspond to energies that mark the edges of "bins", in which there is exactly one bound state. Only for integer $v_{D}$, however, "bin edges" coincide with bound state energies of the potential. In fact, "bin edges" were introduced in this way in Fig. 4 A second useful choice of $v_{0}$ is $v_{0}=v_{\max }+1$. It allows us to define a near threshold vibrational quantum number, which has values $-1,-2,-3, \ldots$ for bound states counting from the top. The near threshold vibrational quantum number for $E=0$ is $v_{D}-v_{\max }-1$, and lies between -1 and 0 . 


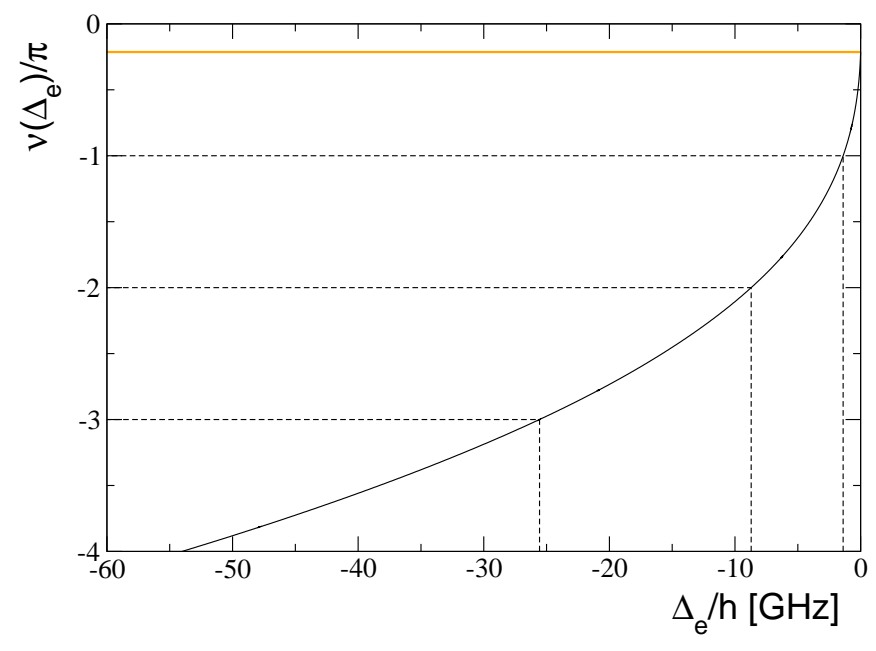

FIG. 10: The near threshold vibrational quantum number $\nu\left(\Delta_{e}\right) / \pi$ of the potential $V\left({ }^{3} \Pi_{u}\right)+\left[2+J_{e}\left(J_{e}+1\right)\right] \hbar^{2} /\left(2 \mu r^{2}\right)$ with $J_{e}=1$ as a function of the binding energy $\Delta_{e}$. The dashed lines indicate the binding energies at which $\nu\left(\Delta_{e}\right) / \pi$ is integer. The value of $\nu\left(\Delta_{e}\right) / \pi$ at $\Delta_{e}=0$ is also indicated.

In practice, for energies far from threshold but not far enough to justify the use of the JWKB approximation the energy dependence of the effective vibrational quantum number can be calculated using expressions given by Mies [64]. Following Refs. [64, 65] we define a near threshold vibrational quantum number $\nu(E) / \pi$ as a continuous function of energy $E$, where $\nu(E)$ is the phase difference between two solutions of the Schrödinger equation $f(r)$ and $\phi(r)$ at the equilibrium separation, $r_{e}$, of the potential $V(r)$. The function $f(r)$ is obtained by solving the Schrödinger equation assuming that $f(0)=0$. The function $\phi(r)$ is obtained by solving the Schrödinger equation assuming that $\phi(\infty)=0$. Then $\tan (\nu(E))$ can be calculated from [64]

$$
\tan (\nu(E))=\frac{\kappa\left(E, r_{e}\right)\left[f\left(r_{e}\right) \phi^{\prime}\left(r_{e}\right)-\phi\left(r_{e}\right) f^{\prime}\left(r_{e}\right)\right]}{\kappa^{2}\left(E, r_{e}\right) f\left(r_{e}\right) \phi\left(r_{e}\right)+\phi^{\prime}\left(r_{e}\right) f^{\prime}\left(r_{e}\right)},
$$

where the primes denote the first derivative with respect to $r$. The near threshold vibrational quantum number, defined this way, has an integer value for energies corresponding to the bound states of the potential $V(r)$.

A connection between the near threshold vibrational quantum number and the binding energies $\Delta_{e}$ of the multichannel excited bound states, used in Fig. 8 can be made using quantum defect theory [64]. The theory states that the shape of the energy dependence of $\nu(E)$ near threshold is nearly independent of the short-range form of the potential and that we can replace the energy $E$ in Eq. C1 by the binding energy $\Delta_{e}$.

The near threshold vibrational quantum number $\nu\left(\Delta_{e}\right) / \pi$ for a potential $V\left({ }^{3} \Pi_{u}\right)+\left[2+J_{e}\left(J_{e}+1\right)\right] \hbar^{2} /\left(2 \mu r^{2}\right)$ as a function of $\Delta_{e}$ is shown in Fig.10] For this potential the short range shape is adjusted to obtain single-channel bound states (or negative integer near threshold vibrational quantum numbers) for binding energies equal to $-1.39 \mathrm{GHz},-8.71 \mathrm{GHz}$, $25.56 \mathrm{GHz}$ and $-54.01 \mathrm{GHz}$. These values agree well with the multi-channel "bin" edges $-1.45 \mathrm{GHz},-8.75 \mathrm{GHz},-25.56$

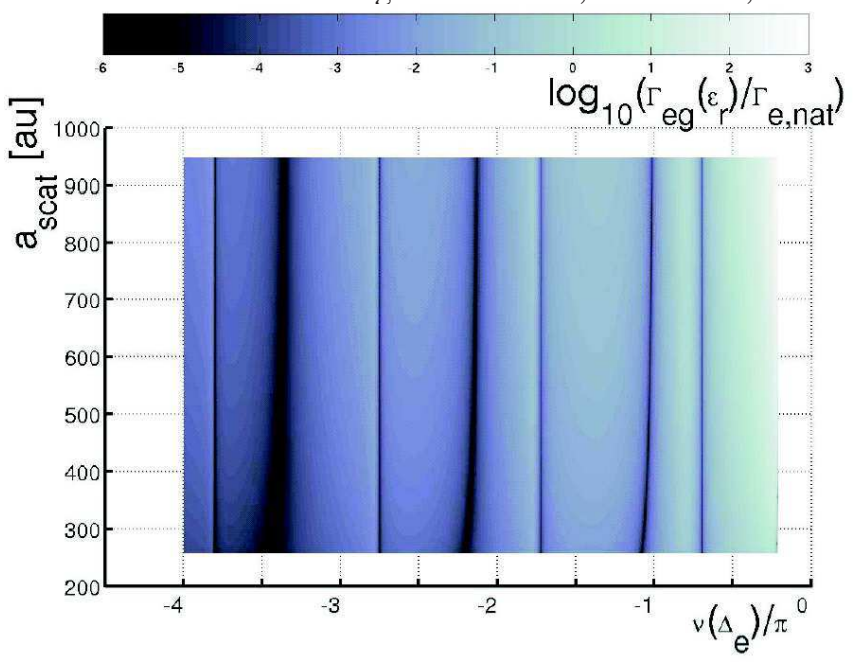

FIG. 11: The stimulated linewidth of the $0_{u}^{+}$band as a function of the near threshold vibrational quantum number $\nu\left(\Delta_{e}\right) / \pi$ and the scattering length $a_{\text {scat }}$ of the ground state potential. The laser intensity is 1 $\mathrm{W} / \mathrm{cm}^{2}$ and the kinetic energy is $\varepsilon_{r} / k_{B}=1 \mu \mathrm{K}$.

GHz and -53.94 GHz shown in Fig. 4 In fact, for binding energies below $-0.026 \mathrm{GHz}$ the multi-channel $0_{u}^{+}$bound states are well approximated by this single-channel Hund's case(c) potential. The connection between integer $\nu\left(\Delta_{e}\right) / \pi$ and the multi-channel bin edges breaks down for smaller binding energies as Coriolis mixing changes the multi-channel coupling scheme from Hund's case (c) to (e). Since a single-channel can not fully simulate the multi-channel bound states, the near threshold vibrational quantum number $\nu\left(\Delta_{e}\right) / \pi=-0.2135$ is not integer for $\Delta_{e}=0$.

Figure 11 shows the results of Fig. 8 in terms of the near threshold vibrational quantum number $\nu\left(\Delta_{e}\right) / \pi$ instead of the binding energy $\Delta_{e}$. It is clearly seen from Fig. [11 that for $\nu\left(\Delta_{e}\right) / \pi$ between $v$ and $v+1$, where $v=-4,-3$, or -2 , and thus for each corresponding bin a pair of dark structures occurs. In fact, the "first kind" of structure always appears at nearly the same location within a bin. The "second kind" of structure moves much more within a bin. Both observations reflect the fact that the $C_{6}$ coefficients of the ground and exited state potentials are similar.
[1] Th. Udem, R. Holzwarth, and T. W. Hänsch, Nature 416, 233 (2002).

[2] L-S Ma, Z. Bi, A. Bartels, L. Robertsson, M. Zucco, R. S.
Windeler, G. Wilpers, C. Oates, L. Hollberg, and S. A. Diddams, Science 303, 1843 (2004).

[3] G. Wilpers, T. Binnewies, C. Degenhardt, U. Sterr, J. Helmcke, 
and F. Riehle, Phys. Rev. Lett. 89, 230801 (2002).

[4] E. A. Curtis, C. W. Oates, L. Hollberg, J. Opt. Soc. Am. B Opt. Phys. 20, 977 (2003).

[5] T. Ido and H. Katori, Phys. Rev. Lett. 91, 053001 (2003).

[6] J. K. Webb, M.T. Murphy, V.V. Flambaum, V. A. Dzuba, J. D. Barrow, C.W. Churchill, J. X. Prochaska, and A. M. Wolfe, Phys. Rev. Lett. 87, 091301 (2001).

[7] Y. Takasu, K. Maki, K. Komori, T. Takano, K. Honda, M. Kumakura, T. Yabuzaki, and Y. Takahashi, Phys. Rev. Lett. 91, 040404 (2003).

[8] R. Maruyama, R. H. Wynar, M. V. Romalis, A. Andalkar, M. D. Swallows, C. E. Pearson, and E. N. Fortson, Phys. Rev. A 68, 011403(R) (2003).

[9] X. Xu, T. H. Loftus, J. W. Dunn, C. H. Greene, J. L. Hall, A. Gallagher, and J. Ye, Phys. Rev. Lett. 90, 193002 (2003).

[10] J. L. Bohn and P. S. Julienne, Phys. Rev. A 60, 414 (1999).

[11] A. Simoni, P. S. Julienne, E. Tiesinga, and C. J. Williams, Phys. Rev. A 66, 063406 (2002).

[12] H. R. Thorsheim, J. Weiner, and P. S. Julienne, Phys. Rev. Lett. 58, 2420 (1987)

[13] P. D. Lett, K. Helmerson, W. D. Phillips, L. P. Ratliff, S. L. Rolston, and M. E. Wagshul, Phys. Rev. Lett. 71, 2200 (1993).

[14] P. S. Julienne, J. Res. Natl. Inst. Stand. Technol. 101, 487 (1996).

[15] J. Weiner, V. S. Baganato, S. Zilio, and P. S. Julienne, Rev. Mod. Phys. 71, 1 (1999).

[16] K. Burnett, P. S. Julienne, P. D. Lett, E. Tiesinga, and C. J. Williams, Nature 416, 225 (2002).

[17] W. D. Phillips, Rev. Mod. Phys. 70, 721 (1998).

[18] K. M. Jones, P. D. Lett, E. Tiesinga, and P. S. Julienne, Phys. Rev. A 61, 012501 (1999).

[19] M. Machholm, P. S. Julienne, and K.-A. Suominen, Phys. Rev. A 64, 033425 (2001).

[20] M. Machholm, P. S. Julienne, and K.-A. Suominen, Phys. Rev. A 65, 023401 (2002).

[21] R. W. Montalvao and R. J. Napolitano, Phys. Rev. A 64, 011403(R) (2001).

[22] E. M. S. Ribeiro, A. L. M Zanelatto, and R. J. Napolitano, Chem. Phys. Lett. 390, 89 (2004).

[23] C. Degenhardt, T. Binnewies, G. Wilpers, U. Sterr, F. Riehle, C. Lisdat, and E. Tiemann, Phys. Rev. A 67, 043408 (2003).

[24] Y. Takahashi, Y. Takasu, K. Maki, K. Komori, T. Takano, K. Honda, A. Yamaguchi, M. Kumakura, and T. Yabuzaki, Laser Phys. 14, 621 (2004).

[25] S. Kotochigova and P. S. Julienne, Potential Energy Surface Database of Group II Dimer Molecules, http://physics.nist.gov/PhysRefData/PES/(2003).

[26] A. Derevianko and S. Porsev, private comunication (2004).

[27] O. Allard, C. Samuelis, A. Pashov, H. Knöckel, and E. Tiemann, Eur. Phys. J. D 26, 155 (2003).

[28] C. Boisseau, E. Audouard, J. Vigué, and P. S. Julienne, Phys. Rev. A 62, 052705 (2000).

[29] E. R. I. Abraham, W. I. McAlexander, C. A. Sackett, and R. G. Hulet, Phys. Rev. Lett. 74, 1315 (1995).

[30] E. Tiesinga, C. J. Williams, P. S. Julienne, K. M. Jones, P. D. Lett, and W. D. Phillips, J. Res. Natl. Inst. Stand. Technol. 101, 505 (1996).

[31] R. Napolitano, J. Weiner, C. J. Williams, and P. S. Julienne, Phys. Rev. Lett. 73, 1352 (1994).

[32] F. H. Mies, Molec. Phys. 41, 953 (1980); 41, 973 (1980).

[33] R. Napolitano, J. Weiner, and P. S. Julienne, Phys. Rev. A 55, 1191 (1997).
[34] M. Harris, E. L. Lewis, D. McHugh, and I. Shannon, J. Phys. B 17, L661 (1984).

[35] A. Bielski, R. Ciuryło, J. Domysławska, D. Lisak, R. S. Trawiński, and J. Szudy, Phys. Rev. A 62, 032511 (2000).

[36] C. MaKenzie, J. H. Denschlag, H. Häffner, A. Browayes, L. E. E. de Araujo, F. K. Fatemi, K. M. Jones, J. E. Simsarian, D. Cho, A. Simoni, E. Tiesinga, P. S. Julienne, K. Helmerson, P. D. Lett, S. L. Rolston, and W. D. Phillips, Phys. Rev. Lett. 88, 120403 (2002).

[37] I. D. Prodan, M. Pichler, M. Junker, R. G. Hulet, and J. L. Bohn, Phys. Rev. Lett. 91, 080402 (2003).

[38] H. T. C. Stoof, A. M. L. Janssen, J. M. V. A. Koelman, and B. J. Verhaar, Phys. Rev. A 39, 3157 (1989).

[39] F. H. Mies, W. J. Stevens, and M. Krauss, J. Mol. Spectrosc. 72, 303 (1978).

[40] E. Czuchaj, M. Krośnicki, and H. Stoll, Theor. Chem. Acc. 110, 28 (2003).

[41] B. Bussery-Honvault, J. M. Launay, and R. Moszynski, Phys. Rev. A 68, 032718 (2003).

[42] S. G. Porsev and A. Derevianko, Phys. Rev. A 65, 020701 (2002).

[43] J. Mitroy and M. W. J. Bromley, Phys. Rev. A 68, 052714 (2003).

[44] G. D. Mahan, J. Chem. Phys. 48, 950 (1968).

[45] G. D. Mahan, J. Chem. Phys. 50, 2755 (1969).

[46] M. Merawa, C. Tendero, and M. Rerat, Chem. Phys. Lett. 343, 397 (2001).

[47] W. L. Wiese, Atomic Spectra Database, http://physics.nist.gov/PhysRefData/ASD1/(2003).

[48] W. J. Meath, J. Chem. Phys. 48, 227 (1968).

[49] E. A. Power, J. Chem. Phys. 46, 4297 (1967).

[50] T. Ido and H. Katori, private comunication (2001).

[51] F. H. Mies, Phys. Rev. A 7, 942 (1973).

[52] B. Gao, Phys. Rev. A 54, 2022 (1996).

[53] R. Drozdowski, J. Kwela, and M. Walkiewicz, Z. Phys. D: At. Mol. Clusters 27, 321 (1993).

[54] B. R. Johnson, J. Chem. Phys. 67, 4086 (1977).

[55] B. R. Johnson, J. Chem. Phys. 69, 4678 (1978).

[56] F. H. Mies, P. S. Julienne and K. M. Sando, Close coupling code, NIST (1988).

[57] O. Dulieu and P. S. Julienne, J. Chem. Phys. 103, 60 (1995).

[58] D. T. Colbert and W. H. Miller, J. Chem. Phys. 96, 1982 (1992).

[59] E. Tiesinga, C. J. Williams, and P. S. Julienne, Phys. Rev. A 57, 4257 (1998)

[60] R. J. LeRoy and R. B. Bernstein, J. Chem. Phys. 52, 3869 (1970).

[61] C. Boisseau, E. Audouard, and J. Vigué, Europhys. Lett. 41, 349 (1998).

[62] G. F. Gribakin and V. V. Flambaum, Phys. Rev. A 48, 546 (1993).

[63] D. Comparat, J. Chem. Phys. 120, 1318 (2004).

[64] F. H. Mies, J. Chem. Phys. 80, 2514 (1984).

[65] F. H. Mies and P. S. Julienne, J. Chem. Phys. 80, 25126 (1984).

[66] It implies a sum over all product states.

[67] In a condensate an extra factor of $1 / 2$ appears in the rate coefficient expression [38].

[68] Excited bound states with even $J_{e}$ and negative parity can predissociate to a scattering state dissociating to the ${ }^{3} P_{0}+{ }^{1} S_{0}$ limit. Such a level can only be excited from $J_{g}=l_{g} \geq 2$ ground scattering states and the excitation probability is negligible at ultracold temperatures on the order of $\mu \mathrm{K}$ and below. 